\title{
Shearlet Smoothness Spaces
}

\author{
Demetrio Labate ${ }^{1}$, Lucia Mantovani ${ }^{2}$ and Pooran $\mathrm{Negi}^{3}$
}

November 27, 2012

\begin{abstract}
The shearlet representation has gained increasingly more prominence in recent years as a flexible mathematical framework which enables the efficient analysis of anisotropic phenomena by combining multiscale analysis with the ability to handle directional information. In this paper, we introduce a class of shearlet smoothness spaces which is derived from the theory of decomposition spaces recently developed by L. Borup and M. Nielsen. The introduction of these spaces is motivated by recent results in image processing showing the advantage of using smoothness spaces associated with directional multiscale representations for the design and performance analysis of improved image restoration algorithms. In particular, we examine the relationship of the shearlet smoothness spaces with respect to Besov spaces, curvelet-type decomposition spaces and shearlet coorbit spaces. With respect to the theory of shearlet coorbit space, the construction of shearlet smoothness spaces presented in this paper does not require the use of a group structure.
\end{abstract}

Key words and phrases: atomic decompositions, Banach frames, Besov spaces, decomposition spaces, shearlets.

AMS Mathematics Subject Classification: 22D10, 42B35, 42C15, 46E35, 47B25.

\section{Introduction}

Over the past twenty years, wavelets and multiscale methods have been extremely successful in applications from harmonic analysis, approximation theory, numerical analysis and image processing. However, it is now well established that, despite their remarkable success, wavelets are not very efficient when dealing with multidimensional functions and signals. This limitation is due to their poor directional sensitivity and limited capability in dealing with the anisotropic features which are frequently dominant in multidimensional applications. To overcome this limitation, a variety of methods have been recently introduced to better capture the geometry of multidimensional data, leading to reformulate wavelet theory and applied Fourier analysis within the setting of an emerging theory of sparse representations. It is indicative of this change of perspective that the latest edition of the classical wavelet textbook by S. Mallat was titled "A wavelet tour of signal processing. The sparse way."

Among the new methods emerged in recent years to overcome the limitations of traditional multiscale systems and wavelets, shearlets, originally introduced by one of the authors and his collaborators in [22], offer a unique combination of very useful properties. Similar to the curvelets of Donoho and Candès [3], the elements of the shearlet system form a pyramid of well localized waveforms ranging not only across various scales and locations, like wavelets, but also at various orientations and with highly anisotropic shapes. This makes the shearlet approach particularly efficient for capturing the anisotropic and directional features of multidimensional data [24]. Thanks to these properties, shearlets provide optimally sparse representations, in a precise sense, for a large class of images and other multidimensional data where wavelets are suboptimal

\footnotetext{
${ }^{1}$ Department of Mathematics, University of Houston, Houston, Texas 77204, USA. E-mail: dlabate@math.uh.edu

2Dipartimento di Matematica, Universita' di Genova, 16146 Genova, ITALY. E-mail: luciamanto@dima.unige.it

${ }^{3}$ Department of Mathematics, University of Houston, Houston, Texas 77204, USA. E-mail: psnegi@math.uh.edu
} 
$[18,20]$. In additions, unlike curvelets and other directional systems recently introduced in the literature, the elements of the shearlet system form an affine-like system whose elements are generated from the action of translation and dilation operators on a finite set of generators. This property provides additional simplicity of construction and a connection with the theory of square group representations of the affine group [7, 23]. Furthermore, shearlets are defined on a Cartesian grid, and this enables a unified framework for continuum and discrete setting which is useful for the digital implementation of shearlets [11].

Many basic questions concerning the study of sparse and efficient representations are closely related to the study of the function spaces associated with these representations. For example, wavelets are 'naturally' associated with Besov spaces, and the notion of sparseness in the wavelet expansion is equivalent to an appropriate smoothness measure in Besov spaces [25]. Similarly, the Gabor systems, which are widely used in time-frequency analysis, are naturally associated to the class of modulation spaces $[1,16]$. In the case of shearlets, a sequence of papers by Dahlke, Kutyniok, Steidl and Teschke [8, 9, 10] have recently introduced a class of shearlet spaces within the framework of the coorbit space theory of Feichtinger and Gröchenig $[14,15]$. This approach exploits the fact that the shearlet transform stems from a square integrable group representation to derive an appropriate notion of shearlet coorbit spaces. In particular, it is shown that all the conditions needed in the general coorbit space theory to obtain atomic decompositions and Banach frames can be satisfied in the new shearlet setting, and that the shearlet coorbit spaces of function on $\mathbb{R}^{2}$ are embedded into Besov spaces.

The goal of this paper is to explore an alternative approach to the construction of smoothness spaces associated with the shearlet representation. Unlike the theory of shearlet coorbit spaces, the approach presented here does not require any group structure and is closely associated with the geometrical properties of the spatial-frequency decomposition of the shearlet construction. Our method is derived from the theory of decomposition spaces originally introduced by Feichtinger and Gröbner $[12,13]$ and recently revisited by Borup and Nielsen [2], who have adapted the theory of decomposition spaces to design a very elegant framework for the construction of smoothness spaces closely associated with particular structured decompositions in the Fourier domain. As will be made clear below, this approach can be viewed as a refinement of the classical construction of Besov spaces, which are associated with the dyadic decomposition of the Fourier space. Beside its mathematical interest, the construction of the shearlet smoothness spaces presented in this paper is also motivated by some recent applications in image restoration where it is shown that the introduction of these smoothness spaces allows one to take advantage of the optimally sparse approximation properties of directional representations such as shearlets and curvelets when dealing with images and other multidimensional data $[26,5]$. In [5] for example, a denoising procedure based on Stein-block thresholding is applied within the class of piecewise $C^{2}$ images away from piecewise $C^{2}$ singularities, a function space which can be precisely described using curvelet or shearlet smoothness spaces. Using this last observation, the authors could derive a minimax estimator for the problem of image denoising outperforming the more traditional wavelet estimator.

The paper is organized as follows. After recalling the basic definitions and results from the theory of decomposition spaces (Section 2) and from the theory of shearlets (Section 3), we introduce the new shearlet decomposition spaces for functions on $\mathbb{R}^{2}$ in Section 4. In particular, we show that there is a Parseval frame forming an atomic decomposition for these spaces and that they are completely characterized by appropriate smoothness conditions on the frame coefficients. We also examine the embeddings of shearlet smoothness spaces into Besov spaces and their relationship with the so-called curvelet spaces, with the systems of shearlet molecules and with the shearlet coorbit spaces mentioned above. Finally, in Section 5 we discuss the extension of these results to higher dimensions.

Remark. After the submission of this manuscript, we became aware of two new papers related to our work. The recent paper by Nielsen and Rasmussen [27] introduces compactly supported frame expansions for decomposition spaces, by modifying the original approach of [2] where the generators of the smoothness spaces were required to be band-limited. One of the main tools of the new method consists in extending the machinery of almost diagonal matrices to Triebel-Lizorkin type spaces. Interestingly, in Section 4.6, we use the almost orthogonality of shearlets (from a work about almost diagonal shearlet decomposition of Fourier Integral Operators by one of us in [19]) to characterize the shearlet smoothness spaces using shearlet 
molecules, a shearlet-like family of functions which are not required to be band-limited. The recent paper by Vera [29] adapts the framwork of Triebel-Lizorking spaces to the shearlet decomposition. Also the paper by Vera uses the construction of smooth Parseval frame of shearlets which we employ in our paper.

\subsection{Notation and definitions}

Before proceeding, it is useful to establish some notation and definitions which are used in the following.

We adopt the convention that $x \in \mathbb{R}^{d}$ is a column vector, i.e., $x=\left(x_{1}, \ldots, x_{d}\right)^{t}$, and that $\xi \in \widehat{\mathbb{R}}^{d}$ (in the frequency domain) is a row vector, i.e., $\xi=\left(\xi_{1}, \ldots, \xi_{d}\right)$. A vector $x$ multiplying a matrix $a \in G L_{d}(\mathbb{R})$ on the right is understood to be a column vector, while a vector $\xi$ multiplying $a$ on the left is a row vector. Thus, $a x \in \mathbb{R}^{d}$ and $\xi a \in \widehat{\mathbb{R}}^{d}$. The Fourier transform of $f \in L^{1}\left(\mathbb{R}^{d}\right)$ is defined as

$$
\hat{f}(\xi)=\int_{\mathbb{R}^{d}} f(x) e^{-2 \pi i \xi x} d x,
$$

where $\xi \in \widehat{\mathbb{R}}^{d}$, and the inverse Fourier transform is

$$
\check{f}(x)=\int_{\widehat{\mathbb{R}}^{d}} f(\xi) e^{2 \pi i \xi x} d \xi .
$$

Recall that a countable collection $\left\{\psi_{i}\right\}_{i \in I}$ in a Hilbert space $\mathcal{H}$ is a Parseval frame (sometimes called a tight frame) for $\mathcal{H}$ if

$$
\sum_{i \in I}\left|\left\langle f, \psi_{i}\right\rangle\right|^{2}=\|f\|^{2}, \quad \text { for all } f \in \mathcal{H} .
$$

This is equivalent to the reproducing formula $f=\sum_{i}\left\langle f, \psi_{i}\right\rangle \psi_{i}$, for all $f \in \mathcal{H}$, where the series converges in the norm of $\mathcal{H}$. Hence a Parseval frame provides a basis-like representation even though it does not need to be a basis in general. We refer the reader to $[4,6]$ for more details about frames.

\section{Decomposition Spaces}

We start by recalling the main facts from the theory of decomposition paces originally introduced by Feichtinger and Gröbner [12,13], which will be used to introduce our new definition of Shearlet Smoothness Spaces in Sec. 4.

\subsection{Coverings in Banach spaces}

A collection $\left\{Q_{i}: i \in I\right\}$ of measurable and bounded sets in $\mathbb{R}^{d}$ is an admissible covering if $\cup_{i \in I} Q_{i}=\mathbb{R}^{d}$, and if there is a $n_{0} \in \mathbb{N}$ such that $\#\left\{j \in I: Q_{i} \cap Q_{j} \neq 0\right\} \leq n_{0}$ for all $i \in I$. Given an admissible covering $\left\{Q_{i}: i \in I\right\}$ of $\mathbb{R}^{d}$, a bounded admissible partition of unity (BAPU) is a family of functions $\Gamma=\left\{\gamma_{i}: i \in I\right\}$ satisfying:

- $\operatorname{supp} \gamma_{i} \subset Q_{i} \quad \forall i \in I$

- $\sum_{i \in I} \gamma_{i}(\xi)=1, \quad \xi \in \mathbb{R}^{d}$,

- $\sup _{i \in I}\left|Q_{i}\right|^{1 / p-1}\left\|\mathcal{F}^{-1} \gamma_{i}\right\|_{L^{p}}<\infty, \quad \forall p \in(0,1]$.

Given $\gamma_{i} \in \Gamma$, let us define the multiplier $\gamma_{i}(D) f=\mathcal{F}^{-1}\left(\gamma_{i} \mathcal{F} f\right), f \in L^{2}\left(\mathbb{R}^{d}\right)$. The conditions in the above definition ensure that $\gamma_{i}(D)$ defines a bounded operator for band-limited functions in $L^{p}\left(\mathbb{R}^{d}\right), 0<p \leq \infty$, uniformly in $i \in I$ (cf. Prop.1.5.1 in [28]).

The following definitions will also be needed. Let $\mathcal{Q}=\left\{Q_{i}: i \in I\right\}$ be an admissible covering. A normed sequence space $Y$ on $I$ is called solid if $b=b_{i} \in Y$ and $\left|a_{i}\right| \leq\left|b_{i}\right|$ for all $i \in I$ implies that $a=a_{i} \in Y$; 
the same space is called $\mathcal{Q}$-regular if $h \in Y$ implies that, for each $i \in I, \tilde{h}(i)=\sum_{j \in \tilde{i}} h(j) \in Y$, with $\tilde{i}:=\left\{j \in I: Q_{i} \cap Q_{j} \neq \emptyset\right\}$; the space is called symmetric if it is invariant under permutations $\rho: I \rightarrow I$.

Let $\mathcal{Q}=\left\{Q_{i}: i \in I\right\}$ be an admissible covering. A strictly positive function $w$ on $\mathbb{R}^{d}$ is called $\mathcal{Q}$ moderate if there exists $C>0$ such that $w(x) \leq C w(y)$ for all $x, y \in Q_{i}$ and all $i \in I$. A strictly positive $\mathcal{Q}$-moderate weight on $I$ (derived from $w$ ) is a sequence $v_{i}=w\left(x_{i}\right), i \in I$, with $x_{i} \in Q_{i}$ and $w$ a $Q$-moderate function.

For a solid (quasi-)Banach sequence space $Y$ on $I$, we define the weighted space $Y_{v}$ as

$$
Y_{v}=\left\{\left\{d_{i}\right\}_{i \in I}:\left\{d_{i} v_{i}\right\}_{i \in I} \in Y\right\} .
$$

Given a subset $J$ of the index set $I$, we use the notation $\widetilde{J}:=\left\{i \in I: \exists j \in J\right.$ s.t. $\left.Q_{i} \cap Q_{j} \neq \emptyset\right\}$. We also define inductively $\widetilde{J}^{(k+1)}:=\widetilde{\widetilde{J}^{(k)}}, k \geq 0$, where we set $\widetilde{J}^{(0)}$ be equal to $J$. Observe that for a fixed index $i \in I$ we have $\tilde{i}:=\left\{j \in I: Q_{i} \cap Q_{j} \neq \emptyset\right\}$. We can now define

$$
{\widetilde{Q_{I}}}^{(k)}:=\bigcup_{j \in \tilde{i}^{(k)}} Q_{j}, \quad \text { and } \quad \widetilde{\gamma}_{i}:=\sum_{j \in \tilde{i}} \gamma_{j}
$$

where $\left\{\gamma_{i}: i \in I\right\}$ is an associated BAPU.

Finally, we introduce the following notion of equivalence for coverings. Let $\mathcal{Q}=\left\{Q_{i}: i \in I\right\}$ and $\mathcal{P}=\left\{P_{h}: h \in H\right\}$ be two admissible coverings. $\mathcal{Q}$ is called subordinate to $\mathcal{P}$ if for every index $i \in I$ there exists $j \in J$ such that $Q_{i} \subset P_{j}$. $\mathcal{Q}$ is called almost subordinate to $\mathcal{P}$, and will be denoted by $\mathcal{Q} \leq \mathcal{P}$, if there exists $k \in \mathbb{N}$ such that $\mathcal{Q}$ is subordinate to $\left\{\widetilde{P}_{j}^{(k)}: j \in J\right\}$. If $\mathcal{Q} \leq \mathcal{P}$ and $\mathcal{P} \leq \mathcal{Q}$, we say that $\mathcal{Q}$ and $\mathcal{P}$ are two equivalent coverings, and we will denote with $\mathcal{Q} \sim \mathcal{P}$. As shown in the next section, this notion is related to a notion of equivalence for functions spaces.

\subsection{Decomposition Spaces and Smoothness spaces}

There is a natural way of defining a function space associated with an admissible covering which was originally introduced in [13]. Specifically, let $\mathcal{Q}=\left\{Q_{i}: i \in I\right\}$ be an admissible covering and $\Gamma$ a corresponding BAPU. Let $Y$ be a solid (quasi-) Banach sequence space on $I$, for which $\ell^{0}(I)$ (the finite sequences on $I$ ) is dense in $Y$. Then, for $p \in(0, \infty]$, the decomposition space $D\left(\mathcal{Q}, L^{p}, Y\right)$ is defined as the set of elements $f \in \mathcal{S}^{\prime}\left(\mathbb{R}^{d}\right)$ such that

$$
\|f\|_{D\left(\mathcal{Q}, L^{p}, Y\right)}=\left\|\left\{\left\|\gamma_{i}(D) f\right\|_{L^{p}}\right\}_{i \in I}\right\|_{Y}<\infty .
$$

It follows from the definition that, for $p \in(0, \infty), \mathcal{S}\left(\mathbb{R}^{d}\right)$ is dense in $D\left(\mathcal{Q}, L^{p}, Y\right)$. Also, one can show that the definition of decomposition space is independent of the particular BAPU, provided that $Y$ is $\mathcal{Q}$-regular [13]. We also have the following important result about the equivalence of decomposition spaces (cf. [2, Theorem $2.11])$.

Theorem 2.1. Let $\mathcal{P}=\left\{P_{i}: i \in I\right\}$ and $\mathcal{Q}=\left\{Q_{j}: j \in J\right\}$ be two equivalent admissible coverings, and $\Gamma=\left\{\gamma_{i}: i \in I\right\}$ and $\Phi=\left\{\phi_{j}: j \in J\right\}$ be corresponding BAPUs. If $\left\{v_{i} ; i \in I\right\}$ and $\left\{u_{j}: j \in J\right\}$ are weights derived from the same moderate function $w$, then

$$
D\left(\mathcal{Q}, L^{p}, Y_{v}\right)=D\left(\mathcal{P}, L^{p}, Y_{u}\right)
$$

with equivalent norms.

In this paper, we are interested in a special class of admissible coverings of the frequency space $\widehat{\mathbb{R}}^{d}$ which are generated from the action of affine maps on an open set. This idea was originally developed in [2] where a detailed treatment can be found. In this section, we will briefly review the aspects of this theory which are useful to derive our results in the following sections. 
Let $\mathcal{T}=\left\{A_{k} \cdot+c_{k}\right\}_{k \in \mathbb{N}}$ be a family of invertible affine transformations on $\widehat{\mathbb{R}}^{d}$ and suppose that there are two bounded open sets $P, Q \in \widehat{\mathbb{R}}^{d}$, with $P$ compactly contained in $Q$ such that the sets $\{Q T: T \in \mathcal{T}\}$ and $\{P T: T \in \mathcal{T}\}$ are admissible coverings. If, in addition, there is a constant $K$ such that

$$
\left(Q A_{k}+c_{k}\right) \cap\left(Q A_{k^{\prime}}+c_{k^{\prime}}\right) \neq 0 \Rightarrow\left\|A_{k^{\prime}}^{-1} A_{k}\right\|_{\ell \infty}<K,
$$

then we call $\mathcal{Q}=\{Q T: T \in \mathcal{T}\}$ a structured admissible covering and $\mathcal{T}$ a structured family of affine transformations. We have the following result:

Proposition 2.2 ([2]). Let $\mathcal{Q}=\{Q T: T \in \mathcal{T}\}$ be a structured admissible covering and $\mathcal{T}$ a structured family of affine transformations. Then there exist:

(a) a BAPU $\left\{\gamma_{T}: T \in \mathcal{T}\right\} \subset \mathcal{S}\left(\widehat{\mathbb{R}}^{d}\right)$ corresponding to $\mathcal{Q}$;

(b) a system $\left\{\phi_{T}: T \in \mathcal{T}\right\} \subset \mathcal{S}\left(\widehat{\mathbb{R}}^{d}\right)$ satisfying:

- $\operatorname{supp} \phi_{T} \subset Q T, \quad \forall T \in \mathcal{T}$,

- $\sum_{T \in \mathcal{T}}\left|\phi_{T}\right|^{2}(\xi)=1, \quad \xi \in \widehat{\mathbb{R}}^{d}$,

- $\sup _{T \in \mathcal{T}}|T|^{1 / p-1}\left\|\mathcal{F}^{-1} \phi_{T}\right\|_{L^{p}}<\infty, \quad \forall p \in(0,1]$.

Note: for $T=(A \cdot+c) \in \mathcal{T}$, we have used the notations $|T|=|\operatorname{det} A|$.

A family of functions fulfilling the three conditions in point $b$ ) of Proposition 2.2 will be called a squared BAPU .

Remark 2.1. Note that, in the case of structured admissible coverings, the characterization of equivalent coverings is simplified. In fact, let $\mathcal{P}=\{P T: T \in \mathcal{T}\}$ and $\mathcal{Q}=\{Q T: T \in \mathcal{T}\}$ be two admissible structured coverings with respect to the same family of transformation $\mathcal{T}$. Then $\mathcal{P} \sim \mathcal{Q}$ if $\# N_{P}<\infty$ and $\# N_{Q}<\infty$, where $N_{P}:=\{T \in \mathcal{T}: P \cap Q T \neq \emptyset\}$ and $N_{Q}:=\{T \in \mathcal{T}: Q \cap P T \neq \emptyset\}$. In fact, that means that $P \subset \bigcup_{T \in N_{P}} Q T$ and $Q \subset \bigcup_{T \in N_{Q}} P T$, hence $P S \subset \bigcup_{T \in N_{P}} Q T S$ and $Q S \subset \bigcup_{T \in N_{Q}} P T S, \forall S \in \mathcal{T}$.

Let $\mathcal{Q}=\{Q T: T \in \mathcal{T}\}$ be a structured admissible covering and $\mathcal{T}$ a structured family of affine transformations. Suppose that $K_{a}$ is a cube in $\widehat{\mathbb{R}}^{d}$ (aligned with the coordinate axes) with side-length $2 a$ satisfying $Q \subset K_{a}$. Corresponding to $K_{a}$, we define the system

$$
\left\{\eta_{n, T}=\left(\phi_{T} e_{n, T}\right)^{\vee}: n \in \mathbb{Z}^{d}, T \in \mathcal{T}\right\},
$$

where

$$
e_{n, T}(\xi)=(2 a)^{-d / 2}|T|^{-1 / 2} \chi_{K_{a}}\left(\xi T^{-1}\right) e^{i \frac{\pi}{a} n \xi T^{-1}}, \quad n \in \mathbb{Z}^{d}, T \in \mathcal{T},
$$

and $\phi_{T}$ is a squared BAPU. The following fact is easy to verify.

Proposition 2.3. The system $\left\{\eta_{n, T}: n \in \mathbb{Z}^{2}, T \in \mathcal{T}\right\}$ is a Parseval frame of $L^{2}\left(\mathbb{R}^{d}\right)$.

When the affine transformations $\mathcal{T}$ are invertible linear transformations (i.e., all translations factors are $c_{k}=0$ ), then the Parseval frame $\left\{\eta_{n, T}\right\}$ is in fact a collection of Meyer-type wavelets. Furthermore, one can go beyond the construction of Parseval frames in $L^{2}\left(\mathbb{R}^{d}\right)$, and use the frame coefficients $\left\{\left\langle f, \eta_{n, T}\right\rangle\right\}$ to characterize the decomposition spaces $D\left(\mathcal{Q}, L^{p}, Y_{v}\right)$. For that, it is useful to introduce the notation:

$$
\eta_{n, T}^{(p)}=|T|^{1 / 2-1 / p} \eta_{n, T} .
$$

We then have the following result from [2]. 
Proposition 2.4. Let $\mathcal{Q}=\{T Q: T \in \mathcal{T}\}$ be a structured admissible covering, $Y$ a solid (quasi-)Banach sequence space on $\mathcal{T}$ and $v$ a $\mathcal{Q}$-moderate weight. For $0<p \leq \infty$ we have the characterization

$$
\|f\|_{D\left(\mathcal{Q}, L^{p}, Y_{v}\right)} \approx\left\|\left\{\left(\sum_{n \in \mathbb{Z}^{d}}\left|\left\langle f, \eta_{n, T}^{(p)}\right\rangle\right|^{p}\right)^{1 / p}\right\}_{T \in \mathcal{T}}\right\|_{Y_{v}} .
$$

Usual modifications apply when $p=\infty$.

Note that the constants in the above characterization are uniform with respect $p \in\left[p_{0}, \infty\right]$ for any $p_{0}>0$.

As Proposition 2.4 indicates, there is a coefficient space associated with the decomposition spaces $D\left(\mathcal{Q}, L^{p}, Y_{v}\right)$. Hence, we define the coefficient space $d\left(\mathcal{Q}, \ell^{p}, Y_{v}\right)$ as the set of coefficients $c=\left\{c_{n, T}: n \in\right.$ $\left.\mathbb{Z}^{d}, T \in \mathcal{T}\right\} \subset \mathbb{C}$, satisfying

$$
\|c\|_{d\left(\mathcal{Q}, \ell^{p}, Y_{v}\right)}=\left\|\left\{\left(\sum_{n \in \mathbb{Z}^{d}}\left|c_{n, T}\right|^{p}\right)^{1 / p}\right\}_{T \in \mathcal{T}}\right\|_{Y_{v}} .
$$

Using this notation, we can define the operators between these spaces. For $f \in D\left(\mathcal{Q}, L^{p}, Y_{v}\right)$ the coefficient operator is the operator $C: D\left(\mathcal{Q}, L^{p}, Y_{v}\right) \rightarrow d\left(\mathcal{Q}, \ell^{p}, Y_{v}\right)$ defined by

$$
C f=\left\{\left\langle f, \eta_{n, T}^{(p)}\right\rangle\right\}_{n, T}
$$

For $\left\{c_{n, T}\right\}_{n, T} \in d\left(\mathcal{Q}, \ell^{p}, Y_{v}\right)$ the reconstruction operator is the mapping $R: d\left(\mathcal{Q}, \ell^{p}, Y_{v}\right) \rightarrow D\left(\mathcal{Q}, L^{p}, Y_{v}\right)$ defined by

$$
R\left\{c_{n, T}\right\}_{n, T}=\sum_{n \in \mathbb{Z}^{d}} c_{n, T} \eta_{n, T}^{(p)}
$$

We have the following result (cf. [2, Thm.2]).

Theorem 2.5. For $0<p \leq \infty$, the coefficient operator and the reconstruction operators are both bounded. This makes $D\left(\mathcal{Q}, L^{p}, Y_{v}\right)$ a retract of $d\left(\mathcal{Q}, \ell^{p}, Y_{v}\right)$, that is, $R C=I d_{D\left(\mathcal{Q}, L^{p}, Y_{v}\right)}$. In particular, we have:

$$
\|f\|_{D\left(\mathcal{Q}, L^{p}, Y_{v}\right)} \approx \inf \left\{\left\|\left\{c_{n, T}\right\}_{n, T}\right\|_{d\left(\mathcal{Q}, \ell^{p}, Y_{v}\right)}: f=\sum_{n, T} c_{n, T}|T|^{\frac{1}{p}-\frac{1}{2}} \eta_{n, T}\right\} .
$$

As a special case of decomposition spaces, let us consider the situation where $\mathcal{T}$ is a structured family of affine transformations, $Y_{v}=\left(\ell^{q}\right)_{v_{w, \beta}}, w$ is a $\mathcal{Q}$-moderate function, $\beta \in \mathbb{R}$ and $v_{w, \beta}=\left\{\left(w\left(b_{T}\right)\right)^{\beta}\right\}_{A_{T} \cdot+b_{T} \in \mathcal{T}}$. In this case, we call the space a Smoothness Space and use the notation:

$$
S_{p, q}^{\beta}(\mathcal{T}, w):=D\left(\mathcal{Q}, L^{p},\left(\ell^{q}\right)_{v_{w, \beta}}\right) .
$$

Let $\left\{\eta_{n, T}\right\}$ be the Meyer-type Parseval frame associated with $\mathcal{T}$, as given by (2.3). By the notation introduced in (2.4), we have that

$$
\left|\left\langle f, \eta_{n, T}^{(\tau)}\right\rangle\right|=|T|^{\frac{1}{p}-\frac{1}{\tau}}\left|\left\langle f, \eta_{n, T}^{(p)}\right\rangle\right|, \quad 0<\tau, p \leq \infty .
$$

Thus, if we assume that there is a $\delta>0$ such that $w\left(b_{T}\right)=w(T) \approx|T|^{1 / \delta}$, for $T \in \mathcal{T}$, then we have that

$$
\begin{aligned}
\|f\|_{S_{p, q}^{\beta}} & \approx\left(\sum_{T \in \mathcal{T}}|T|^{\frac{\beta q}{\delta}}\left(\sum_{n \in \mathbb{Z}^{d}}\left|\left\langle f, \eta_{n, T}^{(p)}\right\rangle\right|^{p}\right)^{q / p}\right)^{1 / q} \\
& \approx\left(\sum_{T \in \mathcal{T}}\left(\sum_{n \in \mathbb{Z}^{d}}\left|\left\langle f, \eta_{n, T}^{(r)}\right\rangle\right|^{p}\right)^{q / p}\right)^{1 / q}, \quad \frac{\beta}{\delta}=\frac{1}{p}-\frac{1}{r}
\end{aligned}
$$


The spaces $S_{p, q}^{\beta}(\mathcal{T}, w)$ provide a natural setting for the analysis of nonlinear approximations. For example, using (2.5), one obtains the Jackson-type inequality:

$$
\inf _{g \in \Sigma_{n}}\|f-g\|_{S_{p, p}^{\beta}} \leq C\|f\|_{S_{\tau, \tau}^{\gamma}} n^{-(\gamma-\beta) / \delta}, \quad \frac{1}{\tau}-\frac{1}{p}=\frac{\gamma-\beta}{\delta}
$$

where

$$
\Sigma_{n}=\left\{g=\sum_{n, T \in \Lambda} c_{n, T} \eta_{n, T}: \# \Lambda \leq n\right\} .
$$

Notice that, using d-dimensional (separable) dyadic wavelets $\left\{\eta_{n, j}\right\}$, with $\mathcal{T}=\left\{2^{j} I_{d}: j \in \mathbb{Z}\right\}$, where $I_{d}$ is the $d$-dimensional identity matrix and $\mathcal{Q}$ is an appropriate structured admissible covering, we obtain that

$$
\|f\|_{S_{p, q}^{\beta}} \approx\left(\sum_{j \in \mathbb{Z}} 2^{j q \frac{d}{2}(\beta / \delta+1 / 2-1 / p)}\left(\sum_{n \in \mathbb{Z}}\left|\left\langle f, \eta_{n, j}\right\rangle\right|^{p}\right)^{q / p}\right)^{1 / q},
$$

which can be identified with the Besov space norm of the Besov space $B_{p, q}^{\frac{\beta}{\delta}}\left(\mathbb{R}^{d}\right)$.

\section{The shearlet representation}

In this section, we recall the construction of the Parseval frames of shearlets in dimension $d=2$.

This construction, which follows the approach in [20,21], produces smooth Parseval frames of shearlets for $L^{2}\left(\mathbb{R}^{2}\right)$ as appropriate combinations of shearlet systems defined in cone-shaped regions of the Fourier domain $\widehat{\mathbb{R}}^{2}$. Hence, we start by partitioning $\widehat{\mathbb{R}}^{2}$ into the following cone-shaped regions:

$$
\mathcal{P}_{1}=\left\{\left(\xi_{1}, \xi_{2}\right) \in \mathbb{R}^{2}:\left|\frac{\xi_{2}}{\xi_{1}}\right| \leq 1\right\}, \mathcal{P}_{2}=\left\{\left(\xi_{1}, \xi_{2}\right) \in \mathbb{R}^{2}:\left|\frac{\xi_{2}}{\xi_{1}}\right|>1\right\} .
$$

To define the shearlet systems associated with these regions, for $\xi=\left(\xi_{1}, \xi_{2}\right) \in \widehat{\mathbb{R}}^{2}$, let $\phi \in C^{\infty}(\mathbb{R})$ be a function such that $\hat{\phi}(\xi) \in[0,1], \operatorname{supp} \hat{\phi} \subset\left[-\frac{1}{8}, \frac{1}{8}\right]$ and $\hat{\phi}=1$ on $\left[-\frac{1}{16}, \frac{1}{16}\right]$ and let also

$$
\widehat{\Phi}(\xi)=\widehat{\Phi}\left(\xi_{1}, \xi_{2}\right)=\hat{\phi}\left(\xi_{1}\right) \hat{\phi}\left(\xi_{2}\right)
$$

and

It follows that

$$
W(\xi)=W\left(\xi_{1}, \xi_{2}\right)=\sqrt{\widehat{\Phi}^{2}\left(2^{-2} \xi_{1}, 2^{-2} \xi_{2}\right)-\widehat{\Phi}^{2}\left(\xi_{1}, \xi_{2}\right)} .
$$

$$
\widehat{\Phi}^{2}\left(\xi_{1}, \xi_{2}\right)+\sum_{j \geq 0} W^{2}\left(2^{-2 j} \xi_{1}, 2^{-2 j} \xi_{2}\right)=1 \text { for }\left(\xi_{1}, \xi_{2}\right) \in \widehat{\mathbb{R}}^{2}
$$

Note that each function $W_{j}^{2}=W^{2}\left(2^{-2 j} \cdot\right), j \geq 0$, has support into the Cartesian corona

$$
C_{j}=\left[-2^{2 j-1}, 2^{2 j-1}\right]^{2} \backslash\left[-2^{2 j-4}, 2^{2 j-4}\right]^{2}
$$

and that the functions $W_{j}^{2}, j \geq 0$, produce a smooth tiling of the frequency plane into Cartesian coronae:

$$
\sum_{j \geq 0} W^{2}\left(2^{-2 j} \xi\right)=1 \text { for } \xi \in \mathbb{R}^{2} \backslash\left[-\frac{1}{8}, \frac{1}{8}\right]^{2} \subset \widehat{\mathbb{R}}^{2} .
$$

Next, let $v \in C^{\infty}(\mathbb{R})$ be chosen so that $\operatorname{supp} v \subset[-1,1]$ and

$$
|v(u-1)|^{2}+|v(u)|^{2}+|v(u+1)|^{2}=1 \quad \text { for }|u| \leq 1
$$


In addition, we will assume that $v(0)=1$ and $v^{(n)}(0)=0$, for all $n \geq 1$. This condition will be required below in the construction of the so-called boundary shearlets.

Hence, for $V_{(1)}\left(\xi_{1}, \xi_{2}\right)=v\left(\frac{\xi_{2}}{\xi_{1}}\right)$ and $V_{(2)}\left(\xi_{1}, \xi_{2}\right)=v\left(\frac{\xi_{1}}{\xi_{2}}\right)$, the shearlet systems associated with the cone-shaped regions $\mathcal{P}_{h}, h=1,2$ are defined as the countable collection of functions

$$
\left\{\psi_{j, \ell, k}^{(h)}: j \geq 0,-2^{j} \leq \ell \leq 2^{j}, k \in \mathbb{Z}^{2}\right\},
$$

where

$$
\hat{\psi}_{j, \ell, k}^{(h)}(\xi)=\left|\operatorname{det} A_{(h)}\right|^{-j / 2} W\left(2^{-j} \xi\right) V_{(h)}\left(\xi A_{(h)}^{-j} B_{(h)}^{-\ell}\right) e^{2 \pi i \xi A_{(h)}^{-j} B_{(h)}^{-\ell} k},
$$

and

$$
A_{(1)}=\left(\begin{array}{ll}
4 & 0 \\
0 & 2
\end{array}\right), \quad B_{(1)}=\left(\begin{array}{ll}
1 & 1 \\
0 & 1
\end{array}\right), \quad A_{(2)}=\left(\begin{array}{ll}
2 & 0 \\
0 & 4
\end{array}\right), \quad B_{(2)}=\left(\begin{array}{ll}
1 & 0 \\
1 & 1
\end{array}\right) .
$$

Notice that the dilation matrices $A_{(1)}, A_{(2)}$ are associated with anisotropic dilations and, more specifically, parabolic scaling dilations; by contrast, the shear matrices $B_{(1)}, B_{(2)}$ are non-expanding and their integer powers control the directional features of the shearlet system. Hence, the systems (3.11) form collections of well-localized functions defined at various scales, orientations and locations, controlled by the indices $j, \ell, k$ respectively. In particular, the functions $\hat{\psi}_{j, \ell, k}^{(1)}$, given by (3.12), are supported inside the trapezoidal regions

$$
\Sigma_{j, \ell}:=\left\{\left(\xi_{1}, \xi_{2}\right): \xi_{1} \in\left[-2^{2 j-1},-2^{2 j-4}\right] \cup\left[2^{2 j-4}, 2^{2 j-1}\right],\left|\frac{\xi_{2}}{\xi_{1}}-\ell 2^{-j}\right| \leq 2^{-j}\right\}
$$

inside the Fourier plane, with a similar condition holding for the functions $\hat{\psi}_{j, \ell, k}^{(2)}$. This is illustrated in Fig. 1.

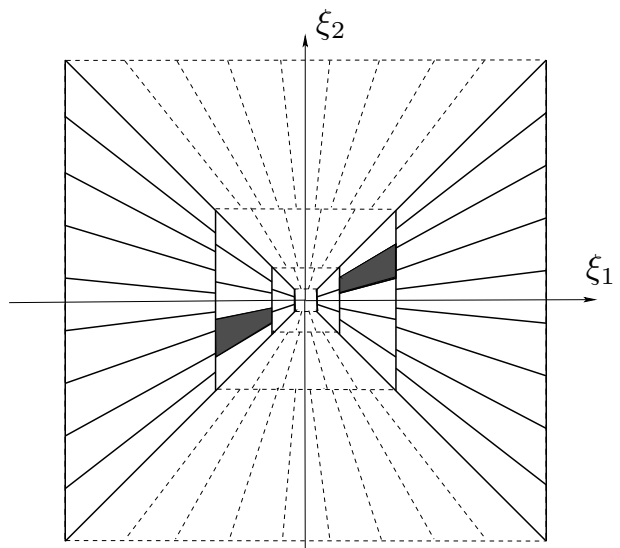

(a)

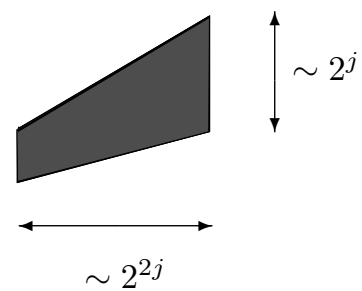

$(b)$

Figure 1: (a) The tiling of the frequency plane $\widehat{\mathbb{R}}^{2}$ induced by the shearlets. (b) Frequency support $\Sigma_{j, \ell}$ of a shearlet $\psi_{j, \ell, k}$, for $\xi_{1}>0$. The other half of the support, for $\xi_{1}<0$, is symmetrical.

As shown in [21], a smooth Parseval frame for $L^{2}\left(\mathbb{R}^{2}\right)$ is obtained by combining the two shearlet systems associated with the cone-based regions $\mathcal{P}_{1}$ and $\mathcal{P}_{2}$ together with a coarse scale system, which takes care of the low frequency region. To ensure that all elements of this combined shearlet system are $C_{c}^{\infty}$ in the frequency domain, the elements whose supports overlap the boundaries of the cone regions in the frequency domain are appropriately modified. Namely the we define shearlet system for $L^{2}\left(\mathbb{R}^{2}\right)$ as the collection

$$
\left\{\widetilde{\psi}_{-1, k}: k \in \mathbb{Z}^{2}\right\} \bigcup\left\{\widetilde{\psi}_{j, \ell, k, h}: j \geq 0,|\ell|<2^{j}, k \in \mathbb{Z}^{2}, h=1,2\right\} \bigcup\left\{\widetilde{\psi}_{j, \ell, k}: j \geq 0, \ell= \pm 2^{j}, k \in \mathbb{Z}^{2}\right\},
$$

consisting of: 
- the coarse-scale shearlets $\left\{\widetilde{\psi}_{-1, k}=\Phi(\cdot-k): k \in \mathbb{Z}^{2}\right\}$, where $\Phi$ is given by (3.7);

- the interior shearlets $\left\{\widetilde{\psi}_{j, \ell, k, h}=\psi_{j, \ell, k}^{(h)}: j \geq 0,|\ell|<2^{j}, k \in \mathbb{Z}^{2}, h=1,2\right\}$, where the functions $\psi_{j, \ell, k}^{(h)}$ are given by (3.12);

- the boundary shearlets $\left\{\widetilde{\psi}_{j, \ell, k}: j \geq 0, \ell= \pm 2^{j}, k \in \mathbb{Z}^{2}\right\}$, obtained by joining together slightly modified versions of $\psi_{j, \ell, k}^{(1)}$ and $\psi_{j, \ell, k}^{(2)}$, for $\ell= \pm 2^{j}$, after that they have been restricted in the Fourier domain to the cones $\mathcal{P}_{1}$ and $\mathcal{P}_{2}$, respectively. The precise definition is given below. For $j=0, k \in \mathbb{Z}^{2}$, $\ell= \pm 1$, we define

$$
\left(\widetilde{\psi}_{0, \ell, k}\right)^{\wedge}(\xi)= \begin{cases}W\left(\xi_{1}, \xi_{2}\right) v\left(\frac{\xi_{2}}{\xi_{1}}-\ell\right) e^{2 \pi i \xi k}, & \text { if } \xi \in \mathcal{P}_{1} \\ W\left(\xi_{1}, \xi_{2}\right) v\left(\frac{\xi_{1}}{\xi_{2}}-\ell\right) e^{2 \pi i \xi k}, & \text { if } \xi \in \mathcal{P}_{2}\end{cases}
$$

For $j \geq 1, \ell= \pm 2^{j}, k \in \mathbb{Z}^{2}$, we define

$$
\left(\widetilde{\psi}_{j, \ell, k}\right)^{\wedge}(\xi)= \begin{cases}2^{-\frac{3}{2} j-\frac{1}{2}} W\left(2^{-2 j} \xi_{1}, 2^{-2 j} \xi_{2}\right) v\left(2^{j} \frac{\xi_{2}}{\xi_{1}}-\ell\right) e^{2 \pi i \xi 2^{-1} A_{(1)}^{-j} B_{(1)}^{-\ell} k}, & \text { if } \xi \in \mathcal{P}_{1} \\ 2^{-\frac{3}{2} j-\frac{1}{2}} W\left(2^{-2 j} \xi_{1}, 2^{-2 j} \xi_{2}\right) v\left(2^{j} \frac{\xi_{1}}{\xi_{2}}-\ell\right) e^{2 \pi i \xi 2^{-1} A_{(1)}^{-j} B_{(1)}^{-\ell} k}, & \text { if } \xi \in \mathcal{P}_{2} .\end{cases}
$$

As detailed in [21], the assumptions on the function $v$ ensure that the boundary shearlets are compactly supported and $C^{\infty}$ in the Fourier domain.

For brevity, let us denote the system (3.15) using the compact notation

$$
\left\{\widetilde{\psi}_{\mu}, \mu \in M\right\}
$$

where $M=M_{C} \cup M_{I} \cup M_{B}$ are the indices associated with coarse scale shearlets, interior shearlets, and boundary shearlets, respectively, given by

- $M_{C}=\left\{\mu=(j, k): j=-1, k \in \mathbb{Z}^{2}\right\}$ (coarse scale shearlets)

- $M_{I}=\left\{\mu=(j, \ell, k, h): j \geq 0,|\ell|<2^{j}, k \in \mathbb{Z}^{2}, h=1,2\right\}$ (interior shearlets)

- $M_{B}=\left\{\mu=(j, \ell, k): j \geq 0, \ell= \pm 2^{j}, k \in \mathbb{Z}^{2}\right\}$ (boundary shearlets).

We have the following result whose proof is essentially the same as in [21]:

Theorem 3.1. The system of shearlets $(3.15)$ is a Parseval frame for $L^{2}\left(\mathbb{R}^{2}\right)$. In addition, the elements of this system are $C^{\infty}$ and compactly supported in the Fourier domain.

Due to the symmetry of the construction, in the following it will be usually sufficient to specialize our to the shearlets in $\mathcal{P}_{1}$. In that case, we will indicate the matrices $A_{(1)}$ and $B_{(1)}$ with $A$ and $B$, and the shearlets in $\mathcal{P}_{1}$ simply with $\psi_{j, \ell, k}$.

\section{Shearlet-type decomposition}

In this section, we define a class of smoothness spaces associated with the shearlet-type decomposition of the frequency plane $\widehat{\mathbb{R}}^{2}$ presented in Section 3 .

\subsection{Shearlet-type covering}

We start by constructing a structured admissible covering of $\widehat{\mathbb{R}}^{2}$ associated with the structured family of affine transformations generating the shearlet systems of Section 3. 
For $A=A_{(1)}$ and $B=B_{(1)}$ given by (3.13), consider the family of affine transformations $\left\{T_{(j, \ell)}:(j, \ell) \in\right.$ $\mathcal{M}$ \} on $\widehat{\mathbb{R}}^{2}$ given by

$$
\xi T_{(j, \ell)}=\xi B^{\ell} A^{j}, \quad(j, \ell) \in \mathcal{M},
$$

where $\mathcal{M}=\left\{(j, \ell) \subset \mathbb{Z} \times \mathbb{Z}^{2}: j \geq 0,|\ell| \leq 2^{j}-1\right\}$. Next, we choose two bounded sets $P$ and $Q$ in $\widehat{\mathbb{R}}^{2}$ defined by $V \cup V^{-}$and $U \cup U^{-}$, respectively, where $V$ is the trapezoid with vertices $(1 / 8,1 / 8),(1 / 2,1 / 2),(1 / 2,-1 / 2)$, $(1 / 8,-1 / 8), V^{-}=\left\{\xi \in \mathbb{R}^{2}:-\xi \in V\right\}, U$ is the trapezoid with vertices $(1 / 16,3 / 16),(9 / 16,11 / 16)$, $(9 / 16,-3 / 16),(9 / 16,-11 / 16)$ and $U^{-}=\left\{\xi \in \mathbb{R}^{2}:-\xi \in U\right\}$. Also, let $U_{0}$ be the cube $[-1 / 2,1 / 2]^{2}, T_{0}$ to be the affine transformation such that $U_{0} \subset P T_{0}$ and $R=\left(\begin{array}{ll}0 & 1 \\ 1 & 0\end{array}\right)$. Hence, let us consider the structured family of affine transformations:

$$
\mathcal{T}_{\mathcal{M}}=\left\{T_{0}, T_{(j, \ell)}, T_{(j, \ell)} R:(j, \ell) \in \mathcal{M}\right\} .
$$

We have the following observation.

Proposition 4.1. The set $\mathcal{Q}=\left\{Q T: T \in \mathcal{T}_{\mathcal{M}}\right\}$, where $\mathcal{T}_{\mathcal{M}}$ is given by (4.18), is a structured admissible covering of $\widehat{\mathbb{R}}^{2}$.

\section{Proof.}

It is easy to verify that:

$$
\widehat{\mathbb{R}}^{2}=U_{0} \cup\left(\bigcup_{(j, \ell) \in \mathcal{M}} P T_{(j, \ell)}\right) \cup\left(\bigcup_{(j, \ell) \in \mathcal{M}} P T_{(j, \ell)} R\right) .
$$

In fact, the right hand side of the above expression describes the shearlet tiling of the frequency plane illustrated in Fig. 1(a). Obviously, the family $\left\{U_{0}, Q T_{(j, \ell)}, Q T_{(j, \ell)} R:(j, \ell) \in \mathcal{M}\right\}$ is also a covering of $\widehat{\mathbb{R}}^{2}$. To conclude that $\left\{Q T: T \in \mathcal{T}_{\mathcal{M}}\right\}$ is a structured admissible covering of $\widehat{\mathbb{R}}^{2}$ we need to prove that the cardinality of the set $\left\{T^{\prime} \in \mathcal{T}_{\mathcal{M}}: Q T \cap Q T \neq \emptyset\right\}$ is globally bounded and that condition (2.2) is satisfied. Due to the symmetry of construction, it is sufficient to specialize our argument to the cone-shaped region $\mathcal{P}_{1}$.

Let us examine the action of a linear mapping $T_{j, \ell} \in \mathcal{T}_{\mathcal{M}}$ on the trapezoid $U$. We have that

$$
U_{(j, \ell)}:=U T_{(j, \ell)}=U\left(\begin{array}{cc}
1 & \ell \\
0 & 1
\end{array}\right)\left(\begin{array}{cc}
2^{2 j} & 0 \\
0 & 2^{j}
\end{array}\right)=U\left(\begin{array}{cc}
2^{2 j} & \ell 2^{j} \\
0 & 2^{j}
\end{array}\right) .
$$

Hence $T_{(j, \ell)}$ maps the trapezoid $U$ into another trapezoid with the action $\left(\xi_{1}, \xi_{2}\right) \mapsto\left(2^{2 j} \xi_{1}, 2^{j}\left(\ell \xi_{1}+\xi_{2}\right)\right)$. Since the projection of $U$ on the first axis is [1/16,9/16], the projections of the trapezoids $U_{(j, \ell)}$ and $U_{\left(j^{\prime}, \ell^{\prime}\right)}$ are $\left[2^{2 j-4}, 92^{2 j-4}\right]$ and $\left[2^{2 j^{\prime}-4}, 92^{2 j^{\prime}-4}\right]$, respectively. Such two intervals intersect if and only if

$$
\begin{array}{ll} 
& 2^{2 j-4} \leq 2^{2 j^{\prime}-4} \leq 92^{2 j-4} \\
\text { or } & 2^{2 j-4} \leq 92^{2 j^{\prime}-4} \leq 92^{2 j-4} \\
\text { or } & 2^{2 j^{\prime}-4} \leq 2^{2 j-4} \leq 92^{2 j-4} \leq 92^{2 j^{\prime}-4} .
\end{array}
$$

The first inequality is satisfied for $j^{\prime}=j, j+1$, the second one for $j^{\prime}=j, j-1$, the third one just for $j^{\prime}=j$. Hence, a fixed trapezoid $U_{(j, \ell)}$ only intersects 3 vertical strips of the form $\left\{\xi_{1} \in\left[2^{2 j^{\prime}-4}, 92^{2 j^{\prime}-4}\right]\right\}$. We want to compute the number of trapezoids intersecting $U_{(j, \ell)}$ in each of those strips. For this reason, in the following the parameters $j$ and $\ell$ are fixed.

To estimate the number of intersections in each strip, we observe that all the trapezoids have the same vertical extension for each $\ell$. This extension is equal to $2^{j}(3 / 16+\ell / 16)-2^{j}(-3 / 16+\ell / 16)=32^{j-3}$ on the left side and $2^{j}(11 / 16+9 \ell / 16)-2^{j}(-11 / 16+9 \ell / 16)=112^{j-3}$ on the right side. We also observe that, once 
we fix $j^{\prime}$ and a value of $\xi_{1}=\bar{\xi}_{1}$, the shear matrix $\left(\begin{array}{ll}1 & 1 \\ 0 & 1\end{array}\right)$ produces a vertical displacement of $2^{-j^{\prime}} \bar{\xi}_{1}$. Indeed we have that

$$
U\left(\begin{array}{ll}
1 & \ell^{\prime} \\
0 & 1
\end{array}\right)\left(\begin{array}{cc}
2^{2 j^{\prime}} & 0 \\
0 & 2^{j^{\prime}}
\end{array}\right)=U\left(\begin{array}{cc}
2^{2 j^{\prime}} & 0 \\
0 & 2^{j^{\prime}}
\end{array}\right)\left(\begin{array}{cc}
1 & 2^{-j^{\prime}} \ell^{\prime} \\
0 & 1
\end{array}\right) .
$$

Hence, the matrix $\left(\begin{array}{cc}1 & \ell^{\prime} \\ 0 & 1\end{array}\right)$ produces a vertical displacement by $2^{-j^{\prime}} \ell^{\prime} \bar{\xi}_{1}$. Further, we know that for $\xi_{1}=$ $2^{2 j^{\prime}-4}$ (the left bound of the trapezoid $U_{\left(j^{\prime}, \ell^{\prime}\right)}$ ) the trapezoid $U_{(j, \ell)}$ has vertical extension $2^{-j+2 j^{\prime}-3}+2^{j-2}$ while the trapezoid $U_{\left(j^{\prime}, \ell^{\prime}\right)}$ is vertically displaced by $2^{-j^{\prime}} 2^{2 j^{\prime}-4}=2^{j^{\prime}-4}$ by each occurrence of $\left(\begin{array}{ll}1 & 1 \\ 0 & 1\end{array}\right)$. To compute the height of $U_{(j, \ell)}$, we write the equations of the lines vertically delimiting the trapezoid as $\xi_{2}=2^{-j}(\ell+1) \xi_{1}+2^{j-3}$ for the highest one, and $\xi_{2}=2^{-j}(\ell-1) \xi_{1}-2^{j-3}$ for the lower one. Then, for those values of $j^{\prime}$ such that $2^{2 j^{\prime}-4}$ is between $2^{2 j-4}$ and $92^{2 j-4}$ (namely, $j^{\prime}=j, j+1$ ), the number of intersections is bounded by

$$
N_{j^{\prime}}=2\left(\frac{2^{2 j^{\prime}-j-3}+2^{j-2}}{2^{j^{\prime}-4}}+1\right)=2\left(2^{j^{\prime}-j+1}+2^{j-j^{\prime}+2}+1\right) .
$$

That is, in the vertical strip $\left[2^{2 j-4}, 92^{2 j-4}\right] \times \mathbb{R}$ there are at most $N_{j}=2\left(2+2^{2}+1\right)=14$ intersections and in the strip $\left[2^{2(j+1)-4}, 92^{2(j+1)-4}\right] \times \mathbb{R}$ there are at most $N_{j+1}=2\left(2^{2}+2^{3}+1\right)=26$ intersections. On the other hand, for $j^{\prime}=j-1$, we have a similar argument: the right side of $U_{j, \ell}$ (that lies in the line $\xi_{1}=92^{2 j^{\prime}-4}$ ) is between $2^{2 j-4}$ and $92^{2 j-4}$. The height of $U_{j, \ell}$ here is $2^{-j}(\ell+1)\left(92^{2 j^{\prime}-4}\right)+2^{j-3}-2^{-j}(\ell-1)\left(92^{2 j^{\prime}-4}\right)+2^{j-3}=$

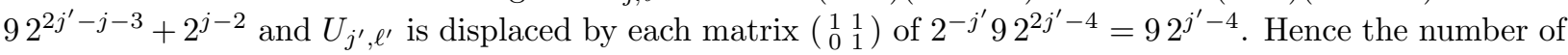
intersections in this strip is bounded by

$$
2\left(\frac{92^{2 j^{\prime}-j-3}+2^{j-2}}{92^{j^{\prime}-4}}+1\right)=2\left(1+2^{3} / 9+1\right) \leq 6 .
$$

when $j^{\prime}=j-1$. Hence, the global number of intersections is controlled by $14+26+6=46$.

It remains to prove property (2.2). First we observe that $U T_{(j, \ell)} \cap U T_{\left(j^{\prime}, \ell^{\prime}\right)} \neq \emptyset$ if and only if $U T_{(j, \ell)} T_{j, \ell}^{-1} \cap$ $U \neq \emptyset$. As above, this happens if $j-j^{\prime} \in\{0, \pm 1\}$. Next we observe that if the intersection of these two sets is not empty, so is the intersection between their second projections. This happens if

$$
\begin{gathered}
-32^{-4} \leq\left(\ell 2^{j-j^{\prime}}-\ell^{\prime} 2^{2\left(j-j^{\prime}\right)}\right) 2^{-4}-32^{j-j^{\prime}-4} \leq 32^{-4} \\
\text { or }-32^{-4} \leq\left(\ell 2^{j-j^{\prime}}-\ell^{\prime} 2^{2\left(j-j^{\prime}\right)}\right) 2^{-4}+32^{j-j^{\prime}-4} \leq 32^{-4} \\
\text { or }\left(\ell 2^{j-j^{\prime}}-\ell^{\prime} 2^{2\left(j-j^{\prime}\right)}\right) 2^{-4}+32^{j-j^{\prime}-4} \geq 32^{-4} \geq\left(\ell 2^{j-j^{\prime}}-\ell^{\prime} 2^{2\left(j-j^{\prime}\right)}\right) 2^{-4}-32^{j-j^{\prime}-4} \geq-32^{-4},
\end{gathered}
$$

indeed $\left(\ell 2^{j-j^{\prime}}-\ell^{\prime} 2^{2\left(j-j^{\prime}\right)}\right) 2^{-4} \pm 32^{j-j^{\prime}-4}$ are the second projections of the points $\left(2^{-4}, 92^{-4}\right) T_{(j, \ell)} T_{\left(j^{\prime}, \ell^{\prime}\right)}$, respectively.

Our goal now is to prove that for each pair $(j, \ell),\left(j^{\prime}, \ell^{\prime}\right) \in \mathcal{M}$ such that $U T_{(j, \ell)} T_{j^{\prime}, \ell^{\prime}}^{-1} \cap U \neq \emptyset$ there exists a constant $K>0$ such that $\left\|T_{(j, \ell)} T_{j^{\prime}, \ell^{\prime}}^{-1}\right\|_{\infty} \leq K$. Since every matrix norm is equivalent, we can use the $\ell^{1}$ norm:

$$
\left\|\left(\begin{array}{cc}
2^{2\left(j-j^{\prime}\right)} & \ell 2^{j-j^{\prime}}-\ell^{\prime} 2^{2\left(j-j^{\prime}\right)} \\
2^{j-j^{\prime}}
\end{array}\right)\right\|_{\ell^{1}}=\max \left\{2^{2\left(j-j^{\prime}\right)},\left|\ell 2^{j-j^{\prime}}-\ell^{\prime} 2^{2\left(j-j^{\prime}\right)}\right|+2^{j-j^{\prime}}\right\} .
$$

Hence we just need a bound for the term $\left|\ell 2^{j-j^{\prime}}-\ell^{\prime} 2^{2\left(j-j^{\prime}\right)}\right|$. We have only three possibilities corresponding to (4.19a), (4.19b) and (4.19c), under the assumption that $j-j^{\prime} \leq 1$. If (4.19a) holds, then we have that

$$
\left|\ell 2^{j-j^{\prime}}-\ell^{\prime} 2^{2\left(j-j^{\prime}\right)}\right| \leq 2^{4}\left|\left(\ell 2^{j-j^{\prime}}-\ell^{\prime} 2^{2\left(j-j^{\prime}\right)}\right) 2^{-4}-32^{j-j^{\prime}-4}\right|+2^{4}\left|32^{j-j^{\prime}-4}\right| \leq 2^{4} 32^{-4}+32=9 ;
$$

if $(4.19 \mathrm{~b})$ holds, then we have that

$$
\left|\ell 2^{j-j^{\prime}}-\ell^{\prime} 2^{2\left(j-j^{\prime}\right)}\right| \leq 2^{4}\left|\left(\ell 2^{j-j^{\prime}}-\ell^{\prime} 2^{2\left(j-j^{\prime}\right)}\right) 2^{-4}+32^{j-j^{\prime}-4}\right| \leq 2^{4} 32^{-3}=3 ;
$$


if (4.19c) holds and if $\ell 2^{j-j^{\prime}}-\ell^{\prime} 2^{2\left(j-j^{\prime}\right)} \geq 0$, we have that

$$
\left|\ell 2^{j-j^{\prime}}-\ell^{\prime} 2^{2\left(j-j^{\prime}\right)}\right| \leq\left(-32^{-4}+32^{j-j^{\prime}-4}\right) 2^{4}=-3+32=3 ;
$$

otherwise, if (4.19c) holds and if $\ell 2^{j-j^{\prime}}-\ell^{\prime} 2^{2\left(j-j^{\prime}\right)} \leq 0$ we have that

$$
\left|\ell 2^{j-j^{\prime}}-\ell^{\prime} 2^{2\left(j-j^{\prime}\right)}\right|=-\ell 2^{j-j^{\prime}}+\ell^{\prime} 2^{2\left(j-j^{\prime}\right)} \leq\left(-32^{-4}+32^{j-j^{\prime}-4}\right) 2^{4} \leq 3 .
$$

In all cases we have that $\left|\ell 2^{j-j^{\prime}}-\ell^{\prime} 2^{2\left(j-j^{\prime}\right)}\right| \leq 9$, and this implies that

$$
\left\|T_{(j, \ell)} T_{\left(j^{\prime}, \ell^{\prime}\right)}^{-1}\right\|_{\ell^{1}} \leq \max \left\{2^{2}, 9+2\right\}=11 .
$$

In the following, we will refer to the structured admissible covering of Proposition 4.1 as the shearlettype covering. By Proposition 2.2, there is at least a BAPU associated with this admissible covering. In Section 4.3 we will analyze the relation between the (Fourier transform of the) Parseval frame of shearlets introducted in Section 3 and one such BAPU.

\subsection{Minimal Admissible Covering}

Given a set $\left\{T_{i}\right\}$ of invertible affine transformations, we say that $\left\{T_{i} Q\right\}$ is a minimal admissible covering if there is no $Q^{\prime}$ s.t. $Q^{\prime}$ is compactly contained in $Q$ and $\left\{T_{i} Q^{\prime}\right\}$ is an admissible covering.

Let us consider the trapezoid

$$
P^{\prime}=\left\{\left(\xi_{1}, \xi_{2}\right):\left|\xi_{2}\right| \leq \xi_{1}, \xi_{1} \in[1 / 8,1 / 2]\right\}
$$

and

$$
P^{\prime \prime}:=P^{\prime} \cup P^{\prime-} .
$$

Proposition 4.2. The set $\left\{P^{\prime \prime} T: T \in \mathcal{T}_{\mathcal{M}}\right\}$, where $\mathcal{T}_{\mathcal{M}}$ is given by (4.18), is a minimal admissible covering in $\widehat{\mathbb{R}}^{2}$. In addition, it is equivalent to any possible structured admissible covering with respect to the family $\mathcal{T}_{\mathcal{M}}$

Proof. Due to the symmetry of the construction, it is sufficient to specialize our argument to the region $\mathcal{P}_{1}^{+}=\left\{\left(\xi_{1}, \xi_{2}\right):\left|\xi_{2}\right| \leq \xi_{1}, \xi_{1} \geq 0\right\}$.

The family of transformations $T_{(j, \ell)},(j, \ell) \in \mathcal{M}$ acts in the following way on $\xi \in \widehat{\mathbb{R}}^{2}$ :

$$
\left(\xi_{1}, \xi_{2}\right) B^{\ell} A^{j}=\left(2^{2 j} \xi_{1}, 2^{j}\left(\ell \xi_{1}+\xi_{2}\right)\right) .
$$

It is easy to verify that, since $T_{(j, \ell)}$ dilates the first coordinate by $2^{2 j}, j \geq 0$, the dilates by $2^{2 j}$ of the interval $[1 / 8,1 / 2)$ cover the set $\xi_{1} \geq 1 / 8$. Note that no interval strictly contained inside $(1 / 8,1 / 2)$ has the same covering property.

Next, note that the action of the shear matrices $B^{\ell}$ consists in shifting by $\ell \xi_{1}$ the second coordinate of each point $\xi_{2}$. In order to cover the truncated cone $\left\{\left(\xi_{1}, \xi_{2}\right):\left|\xi_{2}\right| \leq \xi_{1}, \xi_{1} \geq 1 / 8\right\}$, we need to ensure that such vertical displacement will reach the line $\xi_{2}=\xi_{1}$. Hence, by setting $\ell=2^{j}-1$ which corresponds to the largest vertical displacement, we enforce the condition

$$
2^{j}\left(\left(2^{j}-1\right) \xi_{1}+\xi_{2}\right) \geq 2^{2 j} \xi_{1}
$$

This give the condition $\xi_{2} \geq \xi_{1}$ valid in the first quadrant. This shows that the set $\left\{P^{\prime \prime} T: T \in \mathcal{T}_{\mathcal{M}}\right\}$ is a covering. Furthermore, it is clearly a minimal one.

For the second statement, we need to show that any structured admissible covering with respect to the family of transformation $\mathcal{T}_{\mathcal{M}}$ is equivalent to the covering $\mathcal{P}^{\prime}:=\left\{P^{\prime} T: T \in \mathcal{T}_{\mathcal{M}}\right\}$. That means that, if 
$\mathcal{Q}^{\prime}:=\left\{Q^{\prime} T: T \in \mathcal{T}_{\mathcal{M}}\right\}$ is an admissible structured covering for $\widehat{\mathbb{R}}^{2}$, with $Q^{\prime}$ compact in $\widehat{\mathbb{R}}^{2}$, then $\mathcal{Q}^{\prime}$ and $\mathcal{P}^{\prime}$ are equivalent. Thanks to Theorem 2.1, it is sufficient to prove that $\#\left\{T \in \mathcal{T}_{\mathcal{M}}: P^{\prime} T \cap Q^{\prime} \neq \emptyset\right\}<\infty$ and $\#\left\{T \in \mathcal{T}_{\mathcal{M}}: Q^{\prime} T \cap P^{\prime} \neq \emptyset\right\}<\infty$. Again, we only need to examine the region $\mathcal{P}_{1}^{+}$. Let $\mathcal{Q}^{\prime}:=\left\{Q^{\prime} T: T \in \mathcal{T}_{\mathcal{M}}\right\}$ be an admissible structured covering of $\mathcal{P}_{1}^{+}$, with $Q^{\prime}$ a compact set. Clearly, for $\mathcal{Q}^{\prime}$ to be an admissible structured covering of $\mathcal{P}_{1}^{+}$, it must cover at least the strip domain $\left\{\left(\xi_{1}, \xi_{2}\right): \xi_{1} \in[1 / 8,1 / 2],\left|\xi_{2}\right| \leq \xi_{1}\right\}$. Note that $Q^{\prime}$ cannot contain the origin. Indeed, if $0 \in Q^{\prime}$, then $\forall j \geq 0$ we have that $0 \in Q^{\prime} A^{j}$, hence $\#\left\{j \in \mathbb{N}: Q^{\prime} \cap Q^{\prime} A^{j} \neq \emptyset\right\}=\infty$, which makes $\mathcal{Q}^{\prime}$ non admissible. Thus, we have that $Q^{\prime}$ is contained in $\left\{\left(\xi_{1}, \xi_{2}\right): \xi_{1} \in[m, M],\left|\xi_{2}\right| \leq \xi_{1}\right\}$, with $0<m \leq 1 / 8,1 / 2 \leq M<\infty$. In general, there is a $k \in \mathbb{N}$ so that $M \leq 2^{k}$; that is, $Q^{\prime}$ is contained in finitely many strips of the form $\left\{\left(\xi_{1}, \xi_{2}\right): \xi_{1} \in\left[2^{2 j}, 2^{2 j+1}\right],\left|\xi_{2}\right| \leq \xi_{1}\right\}$. In each of these strips we have a finite number of trapezoids (independently of $j$ ), so that, in conclusion, $Q^{\prime}$ is contained in finitely many sets of the form $P^{\prime} B^{\ell} A^{j}$. For the converse, it is clear that $P^{\prime}$ must be contained in $\tilde{Q}^{\prime}$, since $P^{\prime}$ is associated with a minimal covering.

\subsection{Shearlet Smoothness Spaces}

Having established the existence of a structured admissible covering associated with the shearlet decomposition, we can now define the associated smoothness spaces. Specifically, letting $\mathcal{Q}$ be the shearlet-type covering with $\mathcal{T}_{\mathcal{M}}$ the corresponding family of affine transformations (given in Proposition 4.1) and choosing $w(j, \ell)=2^{j},(j, \ell) \in \mathcal{M}$, to be the $\mathcal{Q}$-moderate weight ${ }^{1}$, the Shearlet Smoothness Spaces are defined by

$$
S_{p, q}^{\beta}\left(\mathcal{T}_{\mathcal{M}}, w\right):=D\left(\mathcal{Q}, L^{p},\left(\ell^{q}\right)_{2^{\beta}}\right) .
$$

As observed above, these spaces are independent from the choice of a particular BAPU.

As indicated at the end of Sec. 2, the dyadic covering of the Fourier space is associated with the Besov spaces. In dimensions $d=2$, let us consider the dyadic partition of the Fourier plane into the Cartesian coronae $\widehat{\mathbb{R}}^{2}=\bigcup_{j \in \mathbb{Z}} C_{j}$, where

$$
C_{j}=\left[-2^{2 j+2}, 2^{2 j+2}\right]^{2} \backslash\left[-2^{2 j}, 2^{2 j}\right]^{2} .
$$

hence, letting $\Omega=\left\{\omega_{j}\right\}_{j \in \mathbb{N}}$ be a partition of unity with supports on the frequency bands (4.20), we have:

$$
\|f\|_{B_{p, q}^{\beta}\left(\mathbb{R}^{2}\right)} \approx\left(\sum_{j \in \mathbb{N}}\left(2^{\beta j}\left\|\omega_{j}(D) f\right\|_{p}\right)^{q}\right)^{1 / q} .
$$

The shearlet-type covering can be considered as a refinement of this covering of $\widehat{\mathbb{R}}^{2}$, suggesting the existence of a close relationship between Besov spaces $B_{p, q}^{\beta}$ and shearlet smoothness spaces. Indeed, we have the following observation which is similar to Lemma 7.4 in [1].

Proposition 4.3. For $0<p \leq \infty, 0<q<\infty$ and $\beta \in \mathbb{R}$ we have

$$
B_{p, q}^{\beta+\frac{1}{q}}\left(\mathbb{R}^{2}\right) \hookrightarrow S_{p, q}^{\beta}\left(\mathbb{R}^{2}\right) .
$$

Likewise:

$$
S_{p, q}^{\beta}\left(\mathbb{R}^{2}\right) \hookrightarrow B_{p, q}^{\beta-s}\left(\mathbb{R}^{2}\right)
$$

where $s=\max (1,1 / p)-\min (1,1 / q)$.

Proof. Let $\left\{\phi_{j, \ell}\right\}$ be a BAPU corresponding to the shearlet-type covering $\mathcal{T}_{\mathcal{M}}$ given in Proposition 4.1. By the properties of the shearlet-like covering, it is clear that we can choose $\Omega$ such that

$$
\operatorname{supp}\left(\phi_{j, \ell}\right) \subset \operatorname{supp}\left(\tilde{\omega}_{j}\right), \ell \in L_{j}, \text { and } \operatorname{supp}\left(\omega_{j}\right) \subset \cup_{\ell \in L_{j}} \operatorname{supp}\left(\tilde{\phi}_{j, l}\right),
$$

\footnotetext{
${ }^{1}$ Here the $\mathcal{Q}$-moderate function $w$ is the first axis projection, and the sequence of points $x_{i} \in Q_{i}$ is given by $x_{i}=\left(2^{j}, 0\right)$.
} 
for all $j \in \mathbb{N}$, where $L_{j}=\left\{\ell:-2^{j} \leq \ell \leq 2^{j}\right\}$. Observe that the cardinality of $L_{j}$ is $2\left(2^{j+1}+1\right)$ (recall that there are 2 sets of transformations associated with the shearlet-type tiling: $T_{(j, \ell)}$ and $\left.T_{(j, \ell)} R\right)$. Thus, for each level $j$ there are about $C 2^{j}$ trapezoids in the covering of $\left\{\phi_{j, l}\right\}$, for some constant $C>0$. It follows that:

$$
\begin{aligned}
\sum_{j \in \mathbb{N}} \sum_{\ell \in L_{j}} 2^{\beta q j}\left\|\phi_{j, \ell}(D) f\right\|_{p}^{q} & =\sum_{j \in \mathbb{N}} \sum_{\ell \in L_{j}} 2^{\beta q j}\left\|\phi_{j, \ell}(D) \tilde{\omega}_{j}(D) f\right\|_{p}^{q} \\
& \leq C \sum_{j \in \mathbb{N}} \sum_{\ell \in L_{j}} 2^{\beta q j}\left\|\tilde{\omega}_{j}(D) f\right\|_{p}^{q} \\
& \leq C \sum_{j \in \mathbb{N}} 2^{j} 2^{\beta q j}\left\|\tilde{\omega}_{j}(D) f\right\|_{p}^{q} .
\end{aligned}
$$

Using a similar calculation we prove the second embedding result in the following four cases.

Case 1 . When $p \geq 1$ and $q<1$ we have:

$$
\begin{aligned}
\sum_{j \in \mathbb{N}}\left(2^{\beta j}\left\|\omega_{j}(D) f\right\|_{p}\right)^{q} & =\sum_{j \in \mathbb{N}}\left(2^{\beta j}\left\|\omega_{j}(D) \sum_{l \in L_{j}} \tilde{\phi}_{j, \ell}(D) f\right\|_{p}\right)^{q} \\
& \leq C \sum_{j \in \mathbb{N}}\left(2^{\beta j}\left\|\sum_{\ell \in L_{j}} \tilde{\phi}_{j, \ell}(D) f\right\|_{p}\right)^{q} \\
& \leq C \sum_{j \in \mathbb{N}}\left(2^{\beta j} \sum_{\ell \in L_{j}}\left\|\tilde{\phi}_{j, \ell}(D) f\right\|_{p}\right)^{q} \\
& \leq C \sum_{j \in \mathbb{N}} \sum_{\ell \in L_{j}}\left(2^{\beta j}\left\|\tilde{\phi}_{j, \ell}(D) f\right\|_{p}\right)^{q} .
\end{aligned}
$$

Case 2. When $p \geq 1$ and $q \geq 1$ we have $s=\left(1-\frac{1}{q}\right)$. As $q \geq 1$, using Hölder's inequality and the fact that cardinality of $L_{j} \sim 2^{j}$ we have:

$$
\begin{aligned}
\sum_{j \in \mathbb{N}}\left(2^{(\beta-s) j}\left\|\omega_{j}(D) f\right\|_{p}\right)^{q} & =\sum_{j \in \mathbb{N}}\left(2^{(\beta-s) j}\left\|\omega_{j}(D) \sum_{l \in L_{j}} \tilde{\phi}_{j, \ell}(D) f\right\|_{p}\right)^{q} \\
& \leq C \sum_{j \in \mathbb{N}}\left(2^{(\beta-s) j}\left\|\sum_{\ell \in L_{j}} \tilde{\phi}_{j, \ell}(D) f\right\|_{p}\right)^{q} \\
& \leq C \sum_{j \in \mathbb{N}}\left(2^{(\beta-s) j} \sum_{\ell \in L_{j}}\left\|\tilde{\phi}_{j, \ell}(D) f\right\|_{p}\right)^{q} \\
& \left.\leq C \sum_{j \in \mathbb{N}} \sum_{\ell \in L_{j}} 2^{\left(\beta-\left(1-\frac{1}{q}\right)\right) j q} 2^{\left(1-\frac{1}{q}\right) j q}\left(\left\|\tilde{\phi}_{j, \ell}(D) f\right\|_{p}\right)^{q} \text { (Hölder's inequality for } q \geq 1\right) \\
& \leq C \sum_{j \in \mathbb{N}} \sum_{\ell \in L_{j}} 2^{\beta j q}\left(\left\|\tilde{\phi}_{j, \ell}(D) f\right\|_{p}\right)^{q}
\end{aligned}
$$

Case 3. When $p<1$ and $q<1$ we have $s=\left(\frac{1}{p}-1\right)$. As $\frac{1}{p}>1$, using Hölder's inequality and the fact 
that $\|\cdot\|_{L_{p}}^{p}$ satisfies the triangle inequality, we have:

$$
\begin{aligned}
\sum_{j \in \mathbb{N}}\left(2^{(\beta-s) j}\left\|\omega_{j}(D) f\right\|_{p}\right)^{q} & =\sum_{j \in \mathbb{N}}\left(2^{(\beta-s) j}\left\|\omega_{j}(D) \sum_{l \in L_{j}} \tilde{\phi}_{j, \ell}(D) f\right\|_{p}\right)^{q} \\
& \leq C \sum_{j \in \mathbb{N}}\left(2^{(\beta-s) j}\left\|\sum_{\ell \in L_{j}} \tilde{\phi}_{j, \ell}(D) f\right\|_{p}\right)^{q} \\
& \leq C \sum_{j \in \mathbb{N}}\left(2^{(\beta-s) j} \sum_{\ell \in L_{j}}\left\|\tilde{\phi}_{j, \ell}(D) f\right\|_{p}^{p}\right)^{\frac{q}{p}} \\
& \leq C \sum_{j \in \mathbb{N}} \sum_{\ell \in L_{j}} 2^{\left(\beta-\left(\frac{1}{p}-1\right)\right) j q} 2^{\left(\frac{1}{p}-1\right) j q}\left(\left\|\tilde{\phi}_{j, \ell}(D) f\right\|_{p}\right)^{q}\left(\text { Hölder's inequality for } \frac{1}{p}>1\right) \\
& \leq C \sum_{j \in \mathbb{N}} \sum_{\ell \in L_{j}} 2^{\beta j q}\left(\left\|\tilde{\phi}_{j, \ell}(D) f\right\|_{p}\right)^{q} .
\end{aligned}
$$

Case 4. When $p<1$ and $q \geq 1$ we have $s=\left(\frac{1}{p}-\frac{1}{q}\right)$. As $\frac{q}{p}>1$, using Hölder's inequality and again the fact that $\|\cdot\|_{L_{p}}^{p}$ satisfies the triangle inequality, we have:

$$
\begin{aligned}
\sum_{j \in \mathbb{N}}\left(2^{(\beta-s) j}\left\|\omega_{j}(D) f\right\|_{p}\right)^{q} & =\sum_{j \in \mathbb{N}}\left(2^{(\beta-s) j}\left\|\omega_{j}(D) \sum_{l \in L_{j}} \tilde{\phi}_{j, \ell}(D) f\right\|_{p}\right)^{q} \\
& \leq C \sum_{j \in \mathbb{N}}\left(2^{(\beta-s) j}\left\|\sum_{\ell \in L_{j}} \tilde{\phi}_{j, \ell}(D) f\right\|_{p}\right)^{q} \\
& \leq C \sum_{j \in \mathbb{N}}\left(2^{(\beta-s) j} \sum_{\ell \in L_{j}}\left\|\tilde{\phi}_{j, \ell}(D) f\right\|_{p}^{p}\right)^{\frac{q}{p}} \\
& \left.\leq C \sum_{j \in \mathbb{N}} \sum_{\ell \in L_{j}} 2^{\left(\beta-\left(\frac{1}{p}-\frac{1}{q}\right)\right) j q} 2^{\left(1-\frac{p}{q}\right) j \frac{q}{p}}\left(\left\|\tilde{\phi}_{j, \ell}(D) f\right\|_{p}\right)^{q} \text { (Hölder's inequality for } \frac{q}{p}>1\right) \\
& \leq C \sum_{j \in \mathbb{N}} \sum_{\ell \in L_{j}} 2^{\left(\beta-\left(\frac{q}{p}-1\right)\right) j} 2^{\left(\frac{q}{p}-1\right) j}\left(\left\|\tilde{\phi}_{j, \ell}(D) f\right\|_{p}\right)^{q} \\
& \leq C \sum_{j \in \mathbb{N}} \sum_{\ell \in L_{j}} 2^{\beta j q}\left(\left\|\tilde{\phi}_{j, \ell}(D) f\right\|_{p}\right)^{q} .
\end{aligned}
$$

\subsection{Equivalence with curvelet spaces}

As mentioned in the introduction, curvelets provide an approach alternative to shearlets for the construction of sparse multidimensional representations. A notion of curvelet spaces is introduced in [1] which is associated with a structured family of affine transformations including rotations and dilations. We can show that the shearlet smoothness spaces defined in Section 4.3 are equivalent to the curvelet spaces.

In order to state our result, let us recall the definition of curvelet covering in dimension two. Note that, in the curvelet covering originally defined in [1], the dilation factors 2 and $\sqrt{2}$ are used, while we used dilations 4 and 2 for the shearlet construction. Both choices provide the same type of parabolic scaling which is needed to ensure the approximation properties of the corresponding representation systems. In order to make shearlet and curvelet tilings 'compatible', in the following we define the curvelet tiling using dilation 
factors 4 and 2. Hence, we define a curvelet covering as the collection of the sets

$$
\begin{aligned}
\left\{S_{j, l}:=\left\{(\rho, \theta) \in \mathbb{R}^{+} \times \mathbb{R}: 2^{2 j-3} \leq \rho \leq 2^{2 j-1}, \theta \in\left[l \frac{2 \pi}{2^{j+1}},(l+1) \frac{2 \pi}{2^{j+1}}\right]\right\}, j \geq 0, l=0, \ldots, 2^{j}-1\right\} \cup \\
\cup\left\{S_{0}:=C_{(0,0)}(1 / 8)\right\}
\end{aligned}
$$

where $C_{(0,0)}(1 / 8)$ is the circle of radius $1 / 8$ centered in $(0,0)$. This covering can be obtained from the family of affine transformations

$$
\mathcal{T}_{C}:=\left\{D_{j, l}: j \geq 0, l=0, \ldots, 2^{j}-1\right\} \cup\left\{D_{0}\right\}
$$

acting on $S_{0,0}$, where $D_{j, l}$ is the affine transformation that brings the element $(\rho, \theta)$ into the element $\left(2^{2 j} \rho, 2^{-j} \theta\right)+c_{j, l}$, with $c_{j, l}=\left(0, l \frac{2 \pi}{2^{j+1}}\right)$ and $D_{0}$ denoting the affine transformation that maps $S_{0}$ in $S_{1,0}$. In fact, $\left\{D_{j, l} S_{0,0}: j \in \mathbb{N}, l=0, \ldots, 2^{j}-1\right\} \cup\left\{D_{0} \tilde{S}_{1,0}\right\}$ is a covering for $\mathbb{R}^{2}$. By construction, the set $S_{0,0}$ is compactly contained in $\tilde{S}_{0,0}$, which is also a covering of $\mathbb{R}^{2}$. Thus we have an admissible structured covering of $\mathbb{R}^{2}$.

Similar to [1], the curvelet smothness spaces are defined as the decomposition spaces which are associated with a curvelet-type covering of $\widehat{\mathbb{R}}^{2}$. We have the following observation.

Proposition 4.4. The shearlet and curvelet smoothness spaces are equivalent with equivalent norms.

Proof. By Theorem 2.1 it is sufficient to show that the curvelet-type covering and the shearlet-type covering are equivalent. Due to the symmetry of construction, it will be sufficient to examine the covering in the region $\mathcal{P}_{1}^{+}$, as done in previous arguments.

Using the notation from Section 4.1, we will show that each trapezoid of the form $Q_{j, \ell}=Q B^{\ell} A^{j}$ has at most a finite number of intersections with the curvelet type tiles $S_{j, l}$ and, vice versa, that each set $S_{j, l}$ has at most a finite number of intersections with the shearlet type tiles $Q_{j, \ell}$, where both numbers are 'global', i.e., independent of $j, l, \ell$. We will ignore intersections occurring at the boundaries of the sets.

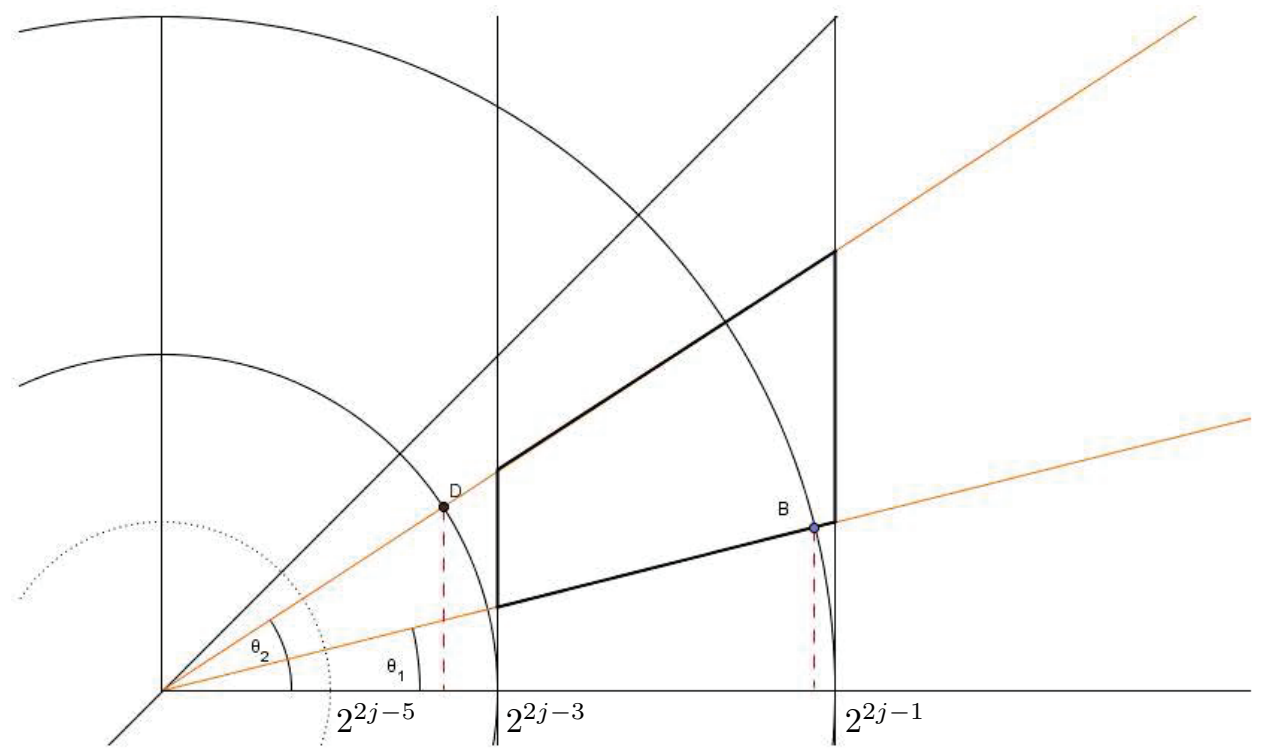

Figure 2: Equivalence of shearlet and curvelet coverings.

As illustrated in Figure 2, any set $S_{j, l}$, at level $j$, extends radially from the value $\cos \left(\theta_{1}\right) 2^{j-1} \leq 2^{2 j-1}$ (i.e., the $\xi_{1}$-coordinate of the point $B$ in the figure) up to $\cos \left(\theta_{2}\right) 2^{2 j-3} \geq 2^{2 j-3} \cos (\pi / 4)=2^{j-3-1 / 2}$ (i.e., the $\xi_{1}$-coordinate of the point $D$ in the figure). Hence, to cover this curvelet-type tile, we need shearlet-type tiles associated with scale parameters ranging from $j-1$ to $j$ (so that the $\xi_{1}$ axis is covered between $2^{2 j-5}$ 
and $\left.2^{2 j-1}\right)$. Next, observe that, at level $j$, any set $S_{j, l}$ (inside $\mathcal{P}_{1}^{+}$) is contained within two lines of slopes $\tan \left(\frac{l 2 \pi}{2^{j+1}}\right)$ and $\tan \left(\frac{(l+1) 2 \pi}{2^{j+1}}\right)$ and that

$$
\left|\tan \left(\frac{l 2 \pi}{2^{j+1}}\right)-\tan \left(\frac{(l+1) 2 \pi}{2^{j+1}}\right)\right|=2^{-j}
$$

Since, for $\xi_{1} \in\left[2^{2 j-3}, 2^{2 j-1}\right]$, the trapezoids $Q_{j, \ell}$ have right height $2^{j}$, it follows that to cover the vertical extension $2^{2 j-1} 2^{-j}=2^{j-1}$ one needs at most 2 such trapezoids. A similar estimate holds for $\xi_{1} \in\left[2^{2 j-5}, 2^{2 j-3}\right]$ so that a total of 4 trapezoids $Q_{j, \ell}$ is sufficient to cover any set $S_{j, l}$ for any $j, l$. Note that the set $S_{0}$ is clearly be covered by the set $Q T_{0}$.

The converse argument is similar. A fixed shearlet-type tile $Q_{j, \ell}$ is supported in the strip-domain $\left\{\xi_{1} \in\right.$ $\left.\left[2^{2 j-3}, 2^{2 j-1}\right]\right\}$, and has left height $2^{j-2}$ and right height $2^{j}$. Inside the polar corona $2^{2 j-3} \leq|\xi| \leq 2^{2 j-1}$, using the observation above, we see that the sets $S_{j, l}$ cover a vertical extension $2^{j-1}$ so that at most 3 such elements are needed to cover $Q_{j, \ell}$. Using the same argument, we have that inside the corona $2^{2 j-5} \leq|\xi| \leq 2^{2 j-3}$ we also need at most 3 sets of $S_{j, l}$. In conclusion, using at most 6 sets $S_{j, l}$ to cover any trapezoid $Q_{j, \ell}$ for any $j, \ell$.

\subsection{Connection with the Parseval frame of shearlets}

Not surprisingly, the systems of shearlets $\left\{\widetilde{\psi}_{\mu}: \mu \in M\right\}$ introduced in Section 3 are closely associated with the shearlet smoothness spaces. Specifically, let us write the elements of the shearlet system in $\mathcal{P}_{1}$, in the Fourier domain, as

$$
\hat{\psi}_{j, \ell, k}(\xi)=\hat{\psi}_{j, \ell}\left(\xi A^{-j} B^{-\ell}\right) u_{j, \ell, k}(\xi),
$$

where $u_{j, \ell, k}(\xi)=|\operatorname{det} A|^{-j / 2} e^{2 \pi i \xi A^{-j} B^{-\ell} k}$ and $\hat{\psi}_{j, \ell}(\xi)=W\left(2^{-j} \xi B^{\ell} A^{j}\right) V(\xi)$. Note that, while $\hat{\psi}_{j, \ell}$ depends on $j, \ell$, its dependence is very mild. In fact, it is easy to see that

$$
\operatorname{supp} \hat{\psi}_{j, \ell} \subset \Sigma_{0,0}=\left\{\left(\xi_{1}, \xi_{2}\right): \xi_{1} \in\left[-2^{-1},-2^{-4}\right] \cup\left[2^{-4}, 2^{-1}\right],\left|\frac{\xi_{2}}{\xi_{1}}\right| \leq 1\right\} \subset\left[\frac{1}{2}, \frac{1}{2}\right]^{2}
$$

and one can show (cf. [21]) that, for each $|\beta| \leq 2 N$, there is a constant $c_{N}$ such that

$$
\left|\frac{\partial^{\beta}}{\partial \xi_{i}}\left(|\xi|^{2 N} \hat{\psi}_{j, \ell}(\xi)\right)\right| \leq c_{N}, \quad i=1,2 .
$$

Similar properties hold for the elements of the shearlet system in $\mathcal{P}_{2}$ and for the boundary shearlets. From these observations it follows (as in [1, Sec.7.3] for curvelets) that, for $0<p \leq \infty$, there are constants $C_{p}, C_{p}^{\prime}$ such that

$$
\sup _{x \in \mathbb{R}^{2}, \ell}\left\|\left\{\psi_{j, \ell, k}(x)\right\}_{k \in \mathbb{Z}^{2}}\right\|_{\ell^{p}} \leq C_{p} 2^{\frac{3}{2} j}, \quad \sup _{k \in \mathbb{Z}^{2}, \ell}\left\|\psi_{j, \ell, k}\right\|_{L^{p}} \leq C_{p}^{\prime} 2^{3 j\left(\frac{1}{2}-\frac{1}{p}\right)},
$$

with appropriate modifications for $p=\infty$.

Using the notation of Section 4.1 we can write each element (4.21) of the shearlet system, in the Fourier domain, as $\hat{\psi}_{j, \ell}\left(\xi T_{(j, \ell)}^{-1}\right) u_{j, \ell, k}(\xi)$, for $T_{(j, \ell)} \in \mathcal{T}_{\mathcal{M}}$ where $\mathcal{T}_{\mathcal{M}}$ is given by (4.18). Clearly, we have that $\left|T_{(j, \ell)}\right|=$ $2^{3 j}$. For simplicity, let us introduce the notation $b_{T_{(j, \ell)}}(\xi)=\left|\hat{\psi}_{j, \ell}\left(\xi T_{(j, \ell)}^{-1}\right)\right|$. Recall that, by the properties discussed in Sec. 3, $\sum_{j \geq 0,|\ell|<2^{j}} b_{T_{(j, \ell)}}^{2}(\xi)=\sum_{j \geq 0,|\ell|<2^{j}}\left|\hat{\psi}_{j, \ell}\left(\xi A^{-j} B^{-\ell}\right)\right|^{2}=1$ on $\left\{\left(\xi_{1}, \xi_{2}\right):\left|\xi_{2}\right| \leq\left|\xi_{1}\right|, \mid \xi_{1}>\right.$ $1 / 8\}$ and that $\widetilde{b}_{T_{(j, \ell)}}^{2}(\xi)=1$ on the set $\Sigma_{j, \ell}$, given by (3.14). Again, as in [1, Sec.7.3], we have that, for $0<p<\infty, b_{T_{(j, \ell)}}(D)$ is a bounded operator on $L^{p}\left(\mathbb{R}^{2}\right)$, uniformly for $j \geq 0$ and ||$\ell \mid<2^{j}$. We hence have the following result which is similar to [2, Lemma 7.5$]$ :

Lemma 4.5. There are constants $C, C^{\prime}<\infty$ such that

$$
\left(\sum_{k \in \mathbb{Z}^{2}}\left|\left\langle f, \psi_{j, \ell, k}\right\rangle\right|^{p}\right)^{1 / p} \leq C\left|T_{(j, \ell)}\right|^{\left(\frac{1}{p}-\frac{1}{2}\right)}\left\|\widetilde{b}_{T_{(j, \ell)}}^{2}(D) f\right\|_{L^{p}}
$$


and

$$
\left\|b_{T_{(j, \ell)}}^{2}(D) f\right\|_{L^{p}} \leq C\left|T_{(j, \ell)}\right|^{\left(\frac{1}{p}-\frac{1}{2}\right)}\left(\sum_{k \in \mathbb{Z}^{2}}\left|\left\langle f, \psi_{j, \ell, k}\right\rangle\right|^{p}\right)^{1 / p}
$$

for all $j \geq 0,|| \ell \mid \leq 2^{j}$.

Proof. For inequality (4.23), note that, for $p \leq 1$ we have:

$$
\begin{aligned}
\sum_{k \in \mathbb{Z}^{2}}\left|\left\langle f, \psi_{j, \ell, k}\right\rangle\right|^{p} & =\sum_{k \in \mathbb{Z}^{2}}\left|\left\langle\widetilde{b}_{T_{(j, \ell)}}^{2}(D) f, \psi_{j, \ell, k}\right\rangle\right|^{p} \leq \sum_{k \in \mathbb{Z}^{2}}\left\|\widetilde{b}_{T_{(j, \ell)}}^{2}(D) f \psi_{j, \ell, k}\right\|_{L^{1}}^{p} \\
& \leq C\left|T_{(j, \ell)}\right|^{1-p} \sum_{k \in \mathbb{Z}^{2}}\left\|\widetilde{b}_{T_{(j, \ell)}}^{2}(D) f \psi_{j, \ell, k}\right\|_{L^{p}}^{p} \leq C^{\prime}\left|T_{(j, \ell)}\right|^{1-\frac{p}{2}}\left\|\widetilde{b}_{T_{(j, \ell)}}^{2}(D) f\right\|_{L^{p}}^{p} .
\end{aligned}
$$

For $1<p<\infty$, using Hölder's inequality and (4.22), we have

$$
\begin{aligned}
\sum_{k \in \mathbb{Z}^{2}}\left|\left\langle f, \psi_{j, \ell, k}\right\rangle\right|^{p} & =\sum_{k \in \mathbb{Z}^{2}}\left|\left\langle\widetilde{b}_{T_{(j, \ell)}}^{2}(D) f, \psi_{j, \ell, k}\right\rangle\right|^{p} \\
& \leq \sup _{x \in \mathbb{R}^{2}} \sum_{k \in \mathbb{Z}^{2}}\left|\psi_{j, \ell, k}(x)\right|^{p}\left\|\widetilde{b}_{T_{(j, \ell)}}^{2}(D) f\right\|_{L^{1}}^{p} \\
& =C\left|T_{(j, \ell)}\right|^{p / 2}\left\|\widetilde{b}_{T_{(j, \ell)}}^{2}(D) f\right\|_{L^{1}}^{p} \\
& \leq C\left|T_{(j, \ell)}\right|^{1-p / 2}\left\|\widetilde{b}_{T_{(j, \ell)}}^{2}(D) f\right\|_{L^{p}}^{p} .
\end{aligned}
$$

For inequality (4.24), note that

$$
\left\|b_{T_{(j, \ell)}}^{2}(D) f\right\|_{L^{p}} \leq C \sum_{\left(j^{\prime}, \ell^{\prime}\right) \in(\tilde{j}, \tilde{\ell})}\left\|\widetilde{b}_{T_{\left(j^{\prime}, \ell^{\prime}\right)}}^{2}(D) f\right\|_{L^{p}} .
$$

For $p \leq 1$, using (4.22), we have:

$$
\begin{aligned}
\left\|\widetilde{b}_{T_{\left(j^{\prime}, \ell^{\prime}\right)}}^{2}(D) f\right\|_{L^{p}}^{p} & =\int_{\mathbb{R}^{2}}\left|\sum_{k \in \mathbb{Z}^{2}}\left\langle f, \psi_{j^{\prime}, \ell^{\prime}, k}\right\rangle \psi_{j^{\prime}, \ell^{\prime}, k}(x)\right|^{p} d x \\
& \leq C \sum_{k \in \mathbb{Z}^{2}}\left|\left\langle f, \psi_{j^{\prime}, \ell^{\prime}, k}\right\rangle\right|^{p} \int_{\mathbb{R}^{2}}\left|\psi_{j^{\prime}, \ell^{\prime}, k}(x)\right|^{p} d x \\
& \leq C\left|T_{\left(j^{\prime}, \ell^{\prime}\right)}\right|^{p / 2-1} \sum_{k \in \mathbb{Z}^{2}}\left|\left\langle f, \psi_{j^{\prime}, \ell^{\prime}, k}\right\rangle\right|^{p} .
\end{aligned}
$$

For $1<p<\infty$, using again Hölder's inequality and (4.22), we have:

$$
\begin{aligned}
\left\|\widetilde{b}_{T_{\left(j^{\prime}, \ell^{\prime}\right)}}^{2}(D) f\right\|_{L^{p}}^{p} & =\int_{\mathbb{R}^{2}}\left|\sum_{k \in \mathbb{Z}^{2}}\left\langle f, \psi_{j^{\prime}, \ell^{\prime}, k}\right\rangle \psi_{j^{\prime}, \ell^{\prime}, k}(x)\right|^{p} d x \\
& \leq \sum_{k \in \mathbb{Z}^{2}}\left|\left\langle f, \psi_{j^{\prime}, \ell^{\prime}, k}\right\rangle\right|^{p} \int_{\Sigma_{j, \ell}}\left(\sum_{k \in \mathbb{Z}^{2}}\left|\psi_{j^{\prime}, \ell^{\prime}, k}(x)\right|^{q}\right)^{p / q} d x \\
& \leq C\left|T_{\left(j^{\prime}, \ell^{\prime}\right)}\right|^{p / 2-1} \sum_{k \in \mathbb{Z}^{2}}\left|\left\langle f, \psi_{j^{\prime}, \ell^{\prime}, k}\right\rangle\right|^{p} .
\end{aligned}
$$

The statement of Lemma 4.5 is valid for the interior shearlets in cone $\mathcal{P}_{1}$. It is easy to see that essentially the same argument holds if one includes also the boundary shearlets. Furthermore, it is clear that a similar 
statement holds for the interior shearlets in the cone $\mathcal{P}_{2}$. Note that, in this case, one need to replace the operators $T_{(j, \ell)}$ with $T_{(j, \ell)} R$, and that also in this case $\left|T_{(j, \ell)} R\right|=\left|T_{(j, \ell)}\right|=2^{3 j}$.

At this point, we can argue as in [2, Sec. 7.3]. Namely, let $\left\{\gamma_{j, \ell}\right\}$ be a BAPU corresponding to the structured family of affine transformations $\mathcal{T}_{\mathcal{M}}$, given by (4.18), associated with the shearlet tiling. We can choose the BAPU so that $\operatorname{supp} \phi_{j, \ell} \subset \operatorname{supp} \widetilde{b}_{T_{j, \ell}}$ and $\operatorname{supp} b_{T_{j, \ell}} \subset \operatorname{supp} \widetilde{\phi}_{j, \ell}$. Hence, by Lemma 4.5 , it follows that

$$
\|f\|_{S_{p, q}^{\beta}} \approx\left(\sum_{h=1}^{2} \sum_{j \geq 0,|\ell| \leq 2^{j}} 2^{j q\left(\beta+3\left(\frac{1}{2}-\frac{1}{p}\right)\right)}\left(\sum_{k \in \mathbb{Z}^{2}}\left|\left\langle f, \psi_{j, \ell, k}^{(h)}\right\rangle\right|^{p}\right)^{q / p}\right)^{1 / q} .
$$

\subsection{Relationship with shearlet molecules}

We have shown that the shearlet smoothness spaces are associated with a special decomposition of the frequency plane and that there are Parseval frames of band-limited functions which provide atomic decompositions of these spaces. A natural question is: are there other types of frame expansions which characterize the same spaces? In this section, we show that is possible to characterize the same spaces using frames of shearlet-like systems which are not necessarily band-limited.

We recall the notion of shearlet molecules from [19]. Let the matrices $A_{(h)}, B_{(h)}, h=1,2$, be as in Section 3 and $\mathcal{N}=\left\{(j, \ell, k): j \geq 0,|\ell| \leq 2^{j}, k \in \mathbb{Z}^{2}\right\}$.

Definition 4.6. For $\mu=(j, \ell, k) \in \mathcal{N}$, the function $m_{\mu}^{(1)}(x)=2^{3 j / 2} a_{\mu}\left(B_{(1)}^{\ell} A_{(1)}^{j} x-k\right)$ is a horizontal shearlet molecule with regularity $R$ if the $a_{\mu}$ satisfies the following properties:

(i) for each $\gamma=\left(\gamma_{1}, \gamma_{2}\right) \in \mathbb{N} \times \mathbb{N}$ and each $N \geq 0$ there is a constant $C_{N}>0$ such that

$$
\left|\partial_{x}^{\gamma} a_{\mu}(x)\right| \leq C_{N}(1+|x|)^{-N}
$$

(ii) for each $M \leq R$ and each $N \geq 0$ there is a constant $C_{N, M}>0$ such that

$$
\left|\hat{a}_{\mu}(\xi)\right| \leq C_{N, M}(1+|\xi|)^{-2 N}\left(2^{-2 j}+\left|\xi_{1}\right|\right)^{M} .
$$

For $\mu=(j, \ell, k) \in \mathcal{N}$, the function $m_{\mu}^{(2)}(x)=2^{3 j / 2} a_{\mu}\left(B_{(2)}^{\ell} A_{(2)}^{j} x-k\right)$ is a vertical shearlet molecule with regularity $R$ if the $\alpha_{\mu}$ satisfies (4.25) and for each $M \leq R$ and each $N \geq 0$ there is a constant $C_{N, M}>0$ such that

$$
\left|\hat{a}_{\mu}(\xi)\right| \leq C_{N, M}(1+|\xi|)^{-2 N}\left(2^{-2 j}+\left|\xi_{2}\right|\right)^{M} .
$$

The constants $C_{N}$ and $C_{N, M}$ are independent of $\mu$.

It was observed in [21] that the systems of shearlets described in Section (3) are special cases of shearlet molecules.

Using the families of horizontal and vertical shearlet molecules, we can use the same idea of the smoothness spaces introduced above to define the following function spaces

$$
\mathfrak{M}_{p, q}^{\beta}=\left\{f \in \mathcal{S}^{\prime}\left(\mathbb{R}^{2}\right): \sum_{h=1}^{2}\left(\sum_{j \geq 0,|\ell| \leq 2^{j}} 2^{j \beta q} 2^{3 j q\left(\frac{1}{2}-\frac{1}{p}\right)}\left(\sum_{k \in \mathbb{Z}^{2}}\left|\left\langle f, \hat{m}_{j, \ell, k}^{(h)}\right\rangle\right|^{p}\right)^{q / p}\right)^{1 / q}<\infty\right\} .
$$

Notice that, unlike the spaces defined above, now the family of function $\left\{m_{\mu}\right\}_{\mu \in \mathcal{M}}$ does not arise from an admissible covering of the plane; indeed, the frequency support of each function $m_{\mu}$ is not required to be compact. We have the following observation 
Proposition 4.7. Given two families of horizontal or vertical shearlet molecules $\left\{m_{\mu}\right\}_{\mu \in \mathcal{N}}$ and $\left\{n_{\mu^{\prime}}\right\}_{\mu^{\prime} \in \mathcal{N}}$, with regularity $R \geq 5$, there exist two constants $C, C^{\prime}$ s.t.

$$
C \sum_{j, \ell, k \in \mathcal{N}} 2^{j \beta q}\left(\left|\left\langle f, n_{(j, \ell, k)}\right\rangle\right|^{p}\right)^{q / p} \leq \sum_{j, \ell, k \in \mathcal{N}} 2^{j \beta q}\left(\left|\left\langle f, m_{(j, \ell, k)}\right\rangle\right|^{p}\right)^{q / p} \leq C^{\prime} \sum_{j, \ell, k \in \mathcal{N}} 2^{j \beta q}\left(\left.\left\langle f, n_{(j, \ell, k)}\right\rangle\right|^{p}\right)^{q / p} .
$$

Proof. Thanks to Proposition 4.8 in [19], we have that $\forall N \in \mathbb{N}$ there exists a constant $C_{N}>0$ s.t.

$$
\left|\left\langle m_{\mu}, n_{\mu^{\prime}}\right\rangle\right| \leq C_{N} \omega\left(\mu, \mu^{\prime}\right)^{-N}
$$

where $R$ is the smallest regularity between the regularities of $m$ and $n$, and $\omega$ is defined as follow:

$$
\omega\left(\mu, \mu^{\prime}\right)=2^{\left|j-j^{\prime}\right|}\left(1+2^{\max \left(j, j^{\prime}\right)} d\left(\mu, \mu^{\prime}\right)\right)
$$

with $d\left(\mu, \mu^{\prime}\right)=\left|\ell 2^{-j}-\ell^{\prime} 2^{-j^{\prime}}\right|^{2}+\left|k_{j, \ell}-k_{j^{\prime}, \ell^{\prime}}^{\prime}\right|^{2}+\left|\left\langle e_{\mu}, k_{j, \ell}-k_{j^{\prime}, \ell^{\prime}}^{\prime}\right\rangle\right|, e_{\mu}=\left(\cos \left(\theta_{\mu}\right), \sin \left(\theta_{\mu}\right)\right)$, and $\theta_{\mu}=$ $\arctan \left(\ell 2^{-j}\right)$.

We also know by [19] that there exists a constant $C>0$ s.t.

$$
\sum_{|\ell| \leq 2^{j}} \sum_{k \in \mathbb{Z}^{2}}\left(1+2^{\gamma} d\left((j, \ell, k),\left(j^{\prime}, \ell^{\prime}, k^{\prime}\right)\right)\right)^{-N} \leq C 2^{2(2 j-\gamma)+},
$$

$\forall N \geq 2$ and $\forall \gamma \in \mathbb{R}$, where $(j-\gamma)_{+}$indicates the positive part. Notice that for each $\gamma \in \mathbb{R}$ we have that $(2 j-\gamma)_{+} \leq|2 j-\gamma|$. Thus we have that

$$
\begin{aligned}
\sum_{\substack{(j, \ell, k) \in \mathcal{N} \\
\left|j-j^{\prime}\right| \geq 1}}\left|\left\langle m_{(j, \ell, k)}, n_{\left(j^{\prime}, \ell^{\prime}, k^{\prime}\right)}\right\rangle\right| & \leq \sum_{\substack{(j, \ell, k) \in \mathcal{N} \\
\left|j-j^{\prime}\right| \geq 1}} C_{N}\left(\omega\left((j, \ell, k),\left(j^{\prime}, \ell^{\prime}, k^{\prime}\right)\right)\right)^{-N} \\
& \leq \sum_{\substack{\left|j-j^{\prime}\right| \geq 1\\
}} C_{N} 2^{-\left|j-j^{\prime}\right| N} \sum_{\substack{|l| \leq 2^{j}-1 \\
k \in \mathbb{Z}}}\left(1+2^{\max \left(j, j^{\prime}\right)} d\left((j, \ell, k),\left(j^{\prime}, \ell^{\prime}, k^{\prime}\right)\right)\right)^{-N} \\
& \leq C_{N} \sum_{\left|j-j^{\prime}\right| \geq 1} 2^{-N\left|j-j^{\prime}\right|} 2^{2\left(2 j-2 \max j, j^{\prime}\right)_{+}} \\
& \leq C_{N} \sum_{\left|j-j^{\prime}\right| \geq 1} 2^{-(N-4)\left|j-j^{\prime}\right|}
\end{aligned}
$$

which is convergent if $N>4$. This condition is satisfied thanks to the assumption that the molecules have regularity $R \geq 5$. Hence, we conclude that there exists a constant $C>0$ s.t.

$$
\sum_{\substack{(j, \ell, k) \in \mathcal{N} \\\left|j-j^{\prime}\right| \geq 1}}\left|\left\langle m_{(j, \ell, k)}, n_{\left(j^{\prime}, \ell^{\prime}, k^{\prime}\right)}\right\rangle\right| \leq C
$$

Since $f \in \mathcal{S}^{\prime}$ and $m_{\mu}, n_{\mu^{\prime}} \in \mathcal{S}$ for all $\mu, \mu^{\prime} \in \mathcal{N}$, we have that there exist two indices $\phi\left(\mu^{\prime}\right), \psi(\mu) \in \mathcal{N}$ s.t.

$$
\begin{gathered}
\left|\left\langle f, n_{\mu^{\prime}}\right\rangle\right| \leq\left|\left\langle f, n_{\phi\left(\mu^{\prime}\right)}\right\rangle\right|, \quad \forall \mu^{\prime} \in \mathcal{N},\left|j-j^{\prime}\right| \geq 1, \\
\left|\left\langle f, n_{\mu^{\prime}}\right\rangle\right|=\left|\left\langle f, n_{\left(j^{\prime}, \ell^{\prime}, k^{\prime}\right)}\right\rangle\right| \leq\left|\left\langle f, n_{\psi(\mu)}\right\rangle\right|, \quad \forall \mu^{\prime}=\left(j^{\prime}, \ell^{\prime}, k^{\prime}\right) \in \mathcal{N} .
\end{gathered}
$$


Combining the above observations, we obtain that

$$
\begin{aligned}
& \left|\left\langle f, m_{(j, \ell, k)}\right\rangle\right| \\
& \leq \sum_{\left(j^{\prime}, \ell^{\prime}, k^{\prime}\right) \in \mathcal{N}}\left|\left\langle f, n_{\left(j^{\prime}, \ell^{\prime}, k^{\prime}\right)}\right\rangle\right|\left|\left\langle m_{(j, \ell, k)}, n_{\left(j^{\prime}, \ell^{\prime}, k^{\prime}\right)}\right\rangle\right| \\
& =\sum_{\substack{\left(j^{\prime}, \ell^{\prime}, k^{\prime}\right) \in \mathcal{N} \\
\left|j-j^{\prime}\right| \geq 1}}\left|\left\langle f, n_{\left(j^{\prime}, \ell^{\prime}, k^{\prime}\right)}\right\rangle\right|\left|\left\langle m_{(j, \ell, k)}, n_{\left(j^{\prime}, \ell^{\prime}, k^{\prime}\right)}\right\rangle\right|+\sum_{\begin{array}{c}
\left(j^{\prime}, \ell^{\prime}, k^{\prime}\right) \in \mathcal{N} \\
\left|j-j^{\prime}\right|=0
\end{array}}\left|\left\langle f, n_{\left(j^{\prime}, \ell^{\prime}, k^{\prime}\right)}\right\rangle\right|\left|\left\langle m_{(j, \ell, k)}, n_{\left(j^{\prime}, \ell^{\prime}, k^{\prime}\right)}\right\rangle\right| \\
& \leq\left|\left\langle f, n_{\phi((j, \ell, k))}\right\rangle\right| \sum_{\substack{\left(j^{\prime}, \ell^{\prime}, k^{\prime}\right) \in \mathcal{N} \\
\left|j-j^{\prime}\right| \geq 1}}\left|\left\langle m_{(j, \ell, k)}, n_{\left(j^{\prime}, \ell^{\prime}, k^{\prime}\right)}\right\rangle\right|+\left|\left\langle f, n_{\psi((j, \ell, k))}\right\rangle\right| \sum_{\substack{\left(j^{\prime}, \ell^{\prime}, k^{\prime}\right) \in \mathcal{N} \\
\left|j-j^{\prime}\right|=0}}\left|\left\langle m_{(j, \ell, k)}, n_{\left(j^{\prime}, \ell^{\prime}, k^{\prime}\right)}\right\rangle\right| \\
& \leq C\left|\left\langle f, n_{\phi((j, \ell, k))}\right\rangle\right|+\left|\left\langle f, n_{\psi((j, \ell, k))}\right\rangle\right| C_{N} C 2^{2(2 j-2 j)} 2^{-N|j-j|} \\
& \leq C_{N}\left|\left\langle f, n_{\xi(\mu)}\right\rangle\right| \text {, }
\end{aligned}
$$

where $\xi(\mu)$ is an element of $\mathcal{N}$ s.t. $\max \left\{\left|\left\langle f, n_{\phi(\mu)}\right\rangle\right|,\left|\left\langle f, n_{\psi(\mu)}\right\rangle\right|\right\} \leq\left|\left\langle f, n_{\xi(\mu)}\right\rangle\right|$

Since the Parseval frames of shearlets are a particular case of shearlet molecules (with regularity $R \geq 5$ ), Prop. 4.7 implies that shearlet smoothness spaces $S_{p, q}^{\beta}$ and the spaces $\mathfrak{M}_{p, q}^{\beta}$ are equivalent with equivalent norms. We refer to [17] for other valuable considerations about the concept of shearlet molecules and the more general notion of parabolic molecules.

\subsection{Relation with coorbit spaces}

In this section, we briefly examine the relationship between the theory of shearlet smoothness spaces presented in this paper and the different approach to the construction of shearlet-type spaces developed by S. Dahlke, G. Kutyniok, G. Steidl, and G. Teschke $[8,9,10]$, which we recall in the following. For $a \in \mathbb{R}^{*}:=\mathbb{R} \backslash\{0\}$ and $s \in \mathbb{R}$, let

$$
M_{a}=\left(\begin{array}{cc}
a & 0 \\
0 & \operatorname{sgn}(a) \sqrt{|a|}
\end{array}\right) \quad \text { and } \quad S_{s}=\left(\begin{array}{cc}
1 & s \\
0 & 1
\end{array}\right) .
$$

The full shearlet group $\mathbb{S}$ is defined as the set $\mathbb{R}^{*} \times \mathbb{R} \times \mathbb{R}^{2}$ endowed with the group operation

$$
(a, s, t)\left(a^{\prime}, s^{\prime}, t^{\prime}\right)=\left(a a^{\prime}, s+s^{\prime} \sqrt{|a|}, t+S_{s} M_{a} t^{\prime}\right) .
$$

A left-invariant Haar measure of $\mathbb{S}$ is given by $\frac{d a}{|a|^{3}} d s d t$ and the shearlet representation is defined as the following unitary representation of the reduced shearlet group $\mathbb{R}^{+} \times \mathbb{R} \times \mathbb{R}^{2}$ :

$$
\pi(a, s, t) g(x)=|a|^{-3 / 4} g\left(M_{a}^{-1} S_{s}^{-1}(x-t)\right) .
$$

We can define the mixed-norm spaces $N_{p, q}$ over the group $G$ by

$$
N_{p, q}:=\left\{\phi:\left(\int_{\mathbb{R}^{*}}\left(\int_{\mathbb{R} \times \mathbb{R}^{2}}|\phi(a, s, t)|^{p} d s d t\right)^{q / p} \frac{d a}{|a|^{3}}\right)^{1 / q}<\infty\right\},
$$

and their weighted versions

$$
N_{p, q, w}:=\left\{\phi:\left(\int_{\mathbb{R}^{*}} w(a)\left(\int_{\mathbb{R} \times \mathbb{R}^{2}}|\phi(a, s, t)|^{p} d s d t\right)^{q / p} \frac{d a}{|a|^{3}}\right)^{1 / q}<\infty\right\}
$$

Note that, in the last definition, we can include the factor $\frac{1}{a^{3}}$ in the weight function $w$ and write the measure simply as $d a$. 
Hence, we define the Mixed Coorbit Shearlet Space as the function space

$$
\mathcal{H}_{p, q, w}:=\left\{f \in \mathcal{S}^{\prime}:\|f\|_{p, q, w}<\infty\right\},
$$

where

$$
\|f\|_{p, q, w}:=\left(\int_{\mathbb{R}^{*}} w(a)\left(\int_{{\mathbb{R} \times \mathbb{R}^{2}}}\left|\left\langle f, \psi_{(a, s, t)}\right\rangle\right|^{p} d s d t\right)^{q / p} d a\right)^{1 / q},
$$

with the elements $\hat{\psi}_{(a, s, t)}=\left(\pi_{(a, s, t)} \psi\right)^{\wedge}$ forming a squared BAPU with respect to shearlet-type tiling.

As discussed in [10], it is sufficient to prove that the space $\mathcal{B}:=\left\{\psi: V_{\psi}(\psi):=<\psi, \pi_{(\cdot)} \psi>\in \mathfrak{W}\left(\mathbb{C}_{0}, L^{1}\right)\right\}$ is not empty to ensure that the space $\mathcal{H}_{p, q, w}$ is well defined. Here $\mathfrak{W}\left(\mathbb{C}_{0}, L^{1}\right)$ the function space $\{F$ : $\left.\left\|L_{x} \chi_{Q} F\right\|_{\infty} \in L^{1}\right\}$, where $\left\|L_{x} \chi_{Q} F\right\|_{\infty}:=\sup _{y \in x Q}|F(y)|$ with $Q$ compact neighbourhood of the identity in $G$. In other words, a function $\psi$ is in $B$ if and only if it satisfies

$$
\begin{gathered}
\left(\int_{\mathbb{R}^{*}} w\left(a_{0}\right)\left(\int_{\mathbb{R}_{\mathbb{R}^{2}}} \sup _{(a, s, t) \in\left(a_{0}, s_{0}, t_{0}\right) Q}\left|V_{\psi} \psi(a, s, t)\right|^{p} d s_{0} d t_{0}\right)^{q / p} d a_{0}\right)^{1 / q}= \\
=\left(\int_{\mathbb{R}^{*}} w\left(a_{0}\right)\left(\int_{\mathbb{R} \times \mathbb{R}^{2}} \sup _{(a, s, t) \in\left(a_{0}, s_{0}, t_{0}\right) Q}|<\psi, \psi(a, s, t)>|^{p} d s_{0} d t_{0}\right)^{q / p} d a_{0}\right)^{1 / q}<\infty .
\end{gathered}
$$

This condition is satisfied, for example, by choosing a function $\psi$ with $\hat{\psi} \in C_{c}^{\infty}$ such as the generator of the shearlet system from Section 3.

Rather than using the continue measures $d a, d s, d t$, we can consider the discrete counting measures on $\mathbb{Z}$ yielding the following space:

$$
\left\{f:\left(\sum_{j} w(j)\left(\sum_{\ell, k}\left|\left\langle f, \psi_{j, \ell, k}\right\rangle\right|^{p}\right)^{q / p}\right)^{1 / q}<\infty\right\} .
$$

This space can be identified with the space $D\left(\mathcal{Q}, L^{p},\left(\ell_{q}\right)_{w}\right)$, where $\mathcal{Q}$ is the tiling obtained by considering dilations, shear transformations and translations on the support of $\psi$. Note however that the shear parameter is ranging over $\mathbb{Z}$ and not over a finite set, as in our shearlet-type covering presented above. Hence, even though we have a decomposition space, this is not equivalent to the smoothness shearlet spaces $S_{p, q}^{\beta}$.

\subsection{Relation with sparsity equivalence}

The following notion of sparsity equivalence was recently introduced in [17].

Definition 4.8. Let $\left\{\phi_{i}: i \in I\right\}$ and $\left\{\psi_{j}: j \in J\right\}$ be two frames of a Hilbert space $\mathcal{H}$ and let $0<p \leq 1$. Then $\left\{\phi_{i}: i \in I\right\}$ and $\left\{\psi_{j}: j \in J\right\}$ are sparsity equivalent in $\ell_{p}$ if, for each $f \in \mathcal{H}$, we have

$$
\left\|\left\{\left\langle f, \phi_{i}\right\rangle\right\}_{i \in I}\right\|_{\ell_{p}}<\infty \Leftrightarrow\left\|\left\{\left\langle f, \psi_{j}\right\rangle\right\}_{j \in J}\right\|_{\ell_{p}}<\infty .
$$

We can observe two important facts, following directly by the 2-dimensional case with the same type of consideration as above:

Theorem 4.9. Let $\mathcal{Q}:=\left\{Q T_{i}: i \in I\right\}$ and $\mathcal{P}:=\left\{P S_{j}: j \in J\right\}$ be two equivalent admissible structured coverings of the space $\mathbb{R}^{d}$, and let $\left\{\phi_{i}: i \in I\right\}$ and $\left\{\psi_{j}: j \in J\right\}$ be two BAPU with respect to $\mathcal{Q}$ and $\mathcal{P}$ respectively. Then, $\left\{\phi_{i}: i \in I\right\}$ and $\left\{\psi_{j}: j \in J\right\}$ are sparsity equivalents. 
Proof. Since $\mathcal{Q}$ and $\mathcal{P}$ are equivalent coverings of the space, for each element $i \in I$ there exists a set $N_{i} \subset J$ such that $\underline{\phi_{i}} \subset \bigcup_{j \in N_{i}} \underline{\psi_{j}}$ and $\phi_{i}=\sum_{j \in \widetilde{N}_{i}} \psi_{j}$, where $\widetilde{N}_{i}$ is the set of indexes $\{j \in J: \exists n \in N$ : $\left.\psi_{j} \cap \psi_{n} \neq \emptyset\right\}$; we also know that $\# \widetilde{N}_{i}$ is finite and does not depend on $i$. For that reason we will denote that cardinality with \# $\widetilde{N}$.

It follows that

$$
\left|\left\langle f, \phi_{i}\right\rangle\right|=\left|\sum_{j \in \widetilde{N}_{i}}\left\langle f, \psi_{j}\right\rangle\right| \leq \sum_{j \in \widetilde{N}_{i}}\left|\left\langle f, \psi_{j}\right\rangle\right| \leq \# \widetilde{N} \max _{\widetilde{N}_{i}}\left|\left\langle f, \psi_{j}\right\rangle\right|=\# \widetilde{N}\left|\left\langle f, \psi_{\eta(j, i)}\right\rangle\right|
$$

for some $\eta(j, i) \in \widetilde{N}_{i}$. It follows that

$$
\left|\left\langle f, \phi_{i}\right\rangle\right|^{p} \leq \# \widetilde{N}^{p}\left|\left\langle f, \psi_{\eta(j, i)}\right\rangle\right|^{p}
$$

and then

$$
\sum_{I}\left|\left\langle f, \phi_{i}\right\rangle\right|^{p} \leq \# \widetilde{N}^{p} \sum_{I}\left|\left\langle f, \psi_{\eta(j, i)}\right\rangle\right|^{p} \leq \# \widetilde{N}^{p} \sum_{J}\left|\left\langle f, \psi_{j}\right\rangle\right|^{p} .
$$

In conclusion, we have that

$$
\left\|\left\{\left\langle f, \psi_{j}\right\rangle\right\}_{j \in J}\right\|_{\ell^{p}}<\infty \Rightarrow\left\|\left\{\left\langle f, \phi_{i}\right\rangle\right\}_{i \in I}\right\|_{\ell^{p}}<\infty .
$$

Using a very similar argument we can prove the converse implication.

We can also observe that in particular decomposition spaces, specifically of the form $D\left(\mathcal{Q}, L^{p}, \ell_{p}\right)$, sparsity equivalence and equivalence of norms are the same. In particular, the equivalence of the norms in the shearlet spaces of the form $\mathcal{S}_{p, p}^{0}$ gives exactly the sparsity equivalence condition.

Let us now consider a couple of families of shearlet molecules $m_{\mu}, n_{\mu}$ as defined in the sections above. From the proof of Theorem 4.7 we know that we have

$$
\left|\left\langle f, m_{\mu}\right\rangle\right| \leq C\left|\left\langle f, n_{\xi(\mu)}\right\rangle\right|
$$

for some constant $C>0$. It then follows that

$$
\sum_{\mu}\left|\left\langle f, m_{\mu}\right\rangle\right|^{p} \leq C \sum_{\nu}\left|\left\langle f, n_{\nu}\right\rangle\right|^{p},
$$

with a similar inequality holding in the opposite direction. Consequently the two families are sparsity equivalent.

Since a family of shearlets is a particular family of shearlet molecules, if follows from the result above that each BAPU associated with an admissible structured covering which is equivalent to the shearlet type tiling is sparsity equivalent to a family of shearlet molecules.

\section{Shearlet-type decompositions in higher dimensions}

The construction of the shearlet smoothness spaces presented above extends to higher dimensions. To illustrate these extension results, it will be sufficient to examine the case $d=3$; other cases are similar.

Similar to the 2-dimensional case, the system of shearlets in $\mathbb{R}^{3}$ are obtained by appropriately combining 3 function systems associated with the pyramidal regions $\mathcal{P}_{1}=\left\{\left(\xi_{1}, \xi_{2}, \xi_{3}\right) \in \widehat{\mathbb{R}}^{3}:\left|\frac{\xi_{2}}{\xi_{1}}\right| \leq 1,\left|\frac{\xi_{3}}{\xi_{1}}\right| \leq 1\right\}, \mathcal{P}_{2}=$ $\left\{\left(\xi_{1}, \xi_{2}, \xi_{3}\right) \in \widehat{\mathbb{R}}^{3}:\left|\frac{\xi_{1}}{\xi_{2}}\right|<1,\left|\frac{\xi_{3}}{\xi_{2}}\right| \leq 1\right\}, \mathcal{P}_{3}=\left\{\left(\xi_{1}, \xi_{2}, \xi_{3}\right) \in \widehat{\mathbb{R}}^{3}:\left|\frac{\xi_{1}}{\xi_{3}}\right|<1,\left|\frac{\xi_{2}}{\xi_{3}}\right|<1\right\}$, in which the Fourier space $\widehat{\mathbb{R}}^{3}$ is partitioned (cf. [21]). Letting $\phi$ be a $C^{\infty}$ univariate function such that $0 \leq \hat{\phi} \leq 1, \hat{\phi}=1$ on $\left[-\frac{1}{16}, \frac{1}{16}\right]$ and $\hat{\phi}=0$ outside the interval $\left[-\frac{1}{8}, \frac{1}{8}\right]$, for $\xi=\left(\xi_{1}, \xi_{2}, \xi_{3}\right) \in \widehat{\mathbb{R}}^{3}$, we define

$$
\widehat{\Phi}(\xi)=\widehat{\Phi}\left(\xi_{1}, \xi_{2}, \xi_{3}\right)=\hat{\phi}\left(\xi_{1}\right) \hat{\phi}\left(\xi_{2}\right) \hat{\phi}\left(\xi_{3}\right)
$$


and $W(\xi)=\sqrt{\widehat{\Phi}^{2}\left(2^{-2} \xi\right)-\widehat{\Phi}^{2}(\xi)}$. It follows that $\widehat{\Phi}^{2}(\xi)+\sum_{j \geq 0} W^{2}\left(2^{-2 j} \xi\right)=1$ for $\xi \in \mathbb{R}^{3}$. Note that each function $W_{j}=W\left(2^{-2 j} \cdot\right), j \geq 0$, is supported inside the Cartesian corona

$$
\left[-2^{2 j-1}, 2^{2 j-1}\right]^{3} \backslash\left[-2^{2 j-4}, 2^{2 j-4}\right]^{3} \subset \widehat{\mathbb{R}}^{3},
$$

and the functions $W^{j}, j \geq 0$, produce a smooth tiling of $\widehat{\mathbb{R}}^{3}$. Next, let $v \in C^{\infty}(\mathbb{R})$ be defined as in Sec. 3 .

For $h=1,2,3, \ell=\left(\ell_{1}, \ell_{2}\right) \in \mathbb{Z}^{2}$, the 3 -dimensional shearlet systems associated with the pyramidal regions $\mathcal{P}_{h}$ are defined as the collections

$$
\left\{\psi_{j, \ell, k}^{(h)}: j \geq 0,-2^{j} \leq \ell_{1}, \ell_{2} \leq 2^{j}, k \in \mathbb{Z}^{3}\right\},
$$

where

$$
\hat{\psi}_{j, \ell, k}^{(h)}(\xi)=\left|\operatorname{det} A_{(d)}\right|^{-j / 2} W\left(2^{-2 j} \xi\right) F_{(h)}\left(\xi A_{(h)}^{-j} B_{(h)}^{[-\ell]}\right) e^{2 \pi i \xi A_{(h)}^{-j} B_{(h)}^{[-\ell]} k},
$$

$F_{(1)}\left(\xi_{1}, \xi_{2}, \xi_{3}\right)=v\left(\frac{\xi_{2}}{\xi_{1}}\right) V\left(\frac{\xi_{3}}{\xi_{1}}\right), F_{(2)}\left(\xi_{1}, \xi_{2}, \xi_{3}\right)=v\left(\frac{\xi_{1}}{\xi_{2}}\right) V\left(\frac{\xi_{3}}{\xi_{2}}\right), F_{(3)}\left(\xi_{1}, \xi_{2}, \xi_{3}\right)=V\left(\frac{\xi_{1}}{\xi_{3}}\right) v\left(\frac{\xi_{2}}{\xi_{3}}\right)$, the anisotropic dilation matrices are given by

$$
A_{(1)}=\left(\begin{array}{lll}
4 & 0 & 0 \\
0 & 2 & 0 \\
0 & 0 & 2
\end{array}\right), A_{(2)}=\left(\begin{array}{lll}
2 & 0 & 0 \\
0 & 4 & 0 \\
0 & 0 & 2
\end{array}\right), A_{(3)}=\left(\begin{array}{lll}
2 & 0 & 0 \\
0 & 2 & 0 \\
0 & 0 & 4
\end{array}\right),
$$

and the shear matrices are defined by

$$
B_{(1)}^{[\ell]}=\left(\begin{array}{ccc}
1 & \ell_{1} & \ell_{2} \\
0 & 1 & 0 \\
0 & 0 & 1
\end{array}\right), B_{(2)}^{[\ell]}=\left(\begin{array}{ccc}
1 & 0 & 0 \\
\ell_{1} & 1 & \ell_{2} \\
0 & 0 & 1
\end{array}\right), B_{(3)}^{[\ell]}=\left(\begin{array}{ccc}
1 & 0 & 0 \\
0 & 1 & 0 \\
\ell_{1} & \ell_{2} & 1
\end{array}\right) .
$$

Note that we have the following relations between the matrices:

$$
B_{(1)}^{[\ell]} A_{1}=R_{z}^{-1} B_{(2)}^{[\ell]} A_{2} R_{z}, \quad B_{(1)}^{[\ell]} A_{1}=R_{y}^{-1} B_{(3)}^{[\ell]} A_{3} R_{y},
$$

where $R_{y}$ and $R_{z}$ denote the rotation with respect to the second and third axes respectively.

Due to the assumptions on $W$ and $v$, the elements of the system of shearlets (5.28) are well localized and band-limited. In particular, the shearlets $\hat{\psi}_{j, \ell, k}^{(1)}(\xi)$ can be written more explicitly as

$$
\hat{\psi}_{j, \ell_{1}, \ell_{2}, k}^{(1)}(\xi)=2^{-j} W\left(2^{-2 j} \xi\right) v\left(2^{j} \frac{\xi_{2}}{\xi_{1}}-\ell_{1}\right) v\left(2^{j} \frac{\xi_{3}}{\xi_{1}}-\ell_{2}\right) e^{2 \pi i \xi A_{(1)}^{-j} B_{(1)}^{\left[-\ell_{1},-\ell_{2}\right]} k},
$$

showing that their supports are contained inside the trapezoidal regions

$$
\left\{\left(\xi_{1}, \xi_{2}, \xi_{3}\right): \xi_{1} \in\left[-2^{2 j-1},-2^{2 j-4}\right] \cup\left[2^{2 j-4}, 2^{2 j-1}\right],\left|\frac{\xi_{2}}{\xi_{1}}-\ell_{1} 2^{-j}\right| \leq 2^{-j},\left|\frac{\xi_{3}}{\xi_{1}}-\ell_{2} 2^{-j}\right| \leq 2^{-j}\right\} .
$$

Similarly for the elements associated with the regions $\mathcal{P}_{2}$ and $\mathcal{P}_{3}$.

Similar to the 2-dimensional case, a Parseval frame of shearlets for $L^{2}\left(\mathbb{R}^{3}\right)$ is obtained by using an appropriate combination of the systems of shearlets associated with the 3 pyramidal regions $\mathcal{P}_{d}, d=1,2,3$, together with a coarse scale system to take care of the low frequency region. Namely, we have:

$$
\begin{aligned}
& \left\{\widetilde{\psi}_{-1, k}: k \in \mathbb{Z}^{3}\right\} \bigcup\left\{\widetilde{\psi}_{j, \ell, k, d}: j \geq 0,\left|\ell_{1}\right|<2^{j},\left|\ell_{2}\right| \leq 2^{j}, k \in \mathbb{Z}^{3}, d=1,2,3\right\} \\
& \bigcup\left\{\widetilde{\psi}_{j, \ell, k}: j \geq 0, \ell_{1}, \ell_{2}= \pm 2^{j}, k \in \mathbb{Z}^{3}\right\}
\end{aligned}
$$

consisting of the coarse-scale shearlets $\left\{\widetilde{\psi}_{-1, k}=\Phi(\cdot-k): k \in \mathbb{Z}^{3}\right\}$; the interior shearlets $\left\{\widetilde{\psi}_{j, \ell, k, d}=\right.$ $\left.\psi_{j, \ell, k}^{(d)}: j \geq 0,\left|\ell_{1}\right|,\left|\ell_{2}\right|<2^{j}, k \in \mathbb{Z}^{3}, d=1,2,3\right\} ;$ the boundary shearlets $\left\{\widetilde{\psi}_{j, \ell, k, d}: j \geq 0,\left|\ell_{1}\right| \leq 2^{j}, \ell_{2}=\right.$ $\left.\pm 2^{j}, k \in \mathbb{Z}^{3}, d=1,2,3\right\}$. The boundary shearlets are obtained by joining together the functions $\psi_{j, \ell, k}^{(h)}$, $h=1,2,3$, for $\ell_{1}= \pm 2^{j}$ or $\ell_{2}= \pm 2^{j}$, after they have been restricted to their respective pyramidal regions (see $[20,21]$ for details). 


\subsection{Shearlet-type covering of $\widehat{\mathbb{R}}^{3}$}

Similar to the 2-dimensional case, we will construct a structured admissible covering of $\widehat{\mathbb{R}}^{3}$ associated with the affine transformations generating the $3 \mathrm{~d}$ shearlet system.

We start by constructing the admissible covering. In $\widehat{\mathbb{R}}^{3}$, let us consider the sets $G_{1}$, with vertices $(1 / 8,1 / 8,1 / 8),(1 / 8,1 / 8,-1 / 8),(1 / 8,-1 / 8,1 / 8)$ and $(1 / 8,-1 / 8,-1 / 8)$, and $G_{2}$, with vertices $(1 / 2,1 / 2,1 / 2)$, $(1 / 2,1 / 2,-1 / 2),(1 / 2,-1 / 2,1 / 2)$ and $(1 / 2,-1 / 2,-1 / 2)$, and let $V$ be the trapezoidal region with the vertical faces given by $G_{1}$ and $G_{2}$. Also, let $V^{-}=\left\{\xi \in \mathbb{R}^{3}:-\xi \in V\right\}$ and $U_{0}$ be the cube $[-1 / 2,1 / 2]^{3}$. Similarly, we define $U$ to be the trapezoidal region with vertical faces given by $G_{1, b}$ and $G_{2, b}$, where $G_{1, b}$ is the vertical square with vertices $(1 / 16,3 / 16,3 / 16),(1 / 16,3 / 16,-3 / 16),(1 / 16,-3 / 16,3 / 16)$ and $(1 / 16,-3 / 16,-3 / 16)$ and $G_{2, b}$ with vertices $(9 / 16,11 / 16,11 / 16),(9 / 16,11 / 16,-11 / 16),(9 / 16,-11 / 16,11 / 16)$ and $(9 / 16,-11 / 16,-11 / 16)$. Again, we set $U^{-}=\left\{\xi \in \mathbb{R}^{3}:-\xi \in U\right\}$. Finally, let $P$ and $Q$ be $V \cup V^{-}$and $U \cup U^{-}$respectively.

For $A_{(1)}$ and $B_{(1)}^{[\ell]}$ given by (5.30) and (5.31), consider the family of affine transformations $\left\{T_{(j, \ell)}:(j, \ell) \in\right.$ $\mathcal{M}$ \} acting on $\widehat{\mathbb{R}}^{3}$ by

$$
\xi T_{(j, \ell)}=\xi T_{j, l}=\xi B_{(1)}^{[\ell]} A_{(1)}^{j}, \quad(j, \ell) \in \mathcal{M},
$$

where $\mathcal{M}=\left\{(j, \ell): j \geq 0,\left|\ell_{1}\right|,\left|\ell_{2}\right| \leq 2^{j}-1\right\}$. Selecting an affine transformation $T_{0}$ such that $U_{0} \subset V T_{0}$, we can define the structured family of affine transformations:

$$
\mathcal{T}_{\mathcal{M}}=\left\{T_{0}, T_{(j, \ell)}, R_{y}^{-1} T_{(j, \ell)} R_{y}, R_{z}^{-1} T_{(j, \ell)} R_{z},(j, \ell) \in \mathcal{M}\right\}
$$

We have the following result.

Proposition 5.1. The set $\mathcal{Q}:=\left\{Q T: T \in \mathcal{T}_{\mathcal{M}}\right\}$, where $\mathcal{T}_{\mathcal{M}}$ is given by (5.34), is a structured admissible covering of $\widehat{\mathbb{R}}^{3}$.

Proof. The argument is similar to the Proposition 4.1.

Direct computation shows that:

$$
\widehat{\mathbb{R}}^{3}=U_{0} \cup\left(\bigcup_{(j, \ell) \in \mathcal{M}} P T_{(j, \ell)}\right) \cup\left(\bigcup_{(j, \ell) \in \mathcal{M}} P R_{y}^{-1} T_{(j, \ell)} R_{y}\right) \cup\left(\bigcup_{(j, \ell) \in \mathcal{M}} P R_{z}^{-1} T_{(j, \ell)} R_{z}\right) .
$$

Since $P$ is compactly contained in $Q$, it is clear that the family $\left\{U_{0}, Q R_{y}^{-1} T_{(j, \ell)} R_{y}, Q R_{z}^{-1} T_{(j, \ell)} R_{z}:(j, \ell) \in \mathcal{M}\right\}$ is also a covering of $\widehat{\mathbb{R}}^{3}$. For $\left\{Q T: T \in \mathcal{T}_{\mathcal{M}}\right\}$ to be a structured admissible covering of $\widehat{\mathbb{R}}^{3}$, we must prove that condition $(2.2)$ is satisfied. Due to the symmetry of the construction, it will be sufficient to show that the sets $\left\{Q T_{0}, Q T_{(j, \ell)}:(j, \ell) \in \mathcal{M}\right\}$ cover the pyramidal region $\mathcal{P}_{1}$.

We have that

$$
Q_{(j, \ell)}:=Q B_{(1)}^{[\ell]} A_{(1)}^{j}=Q\left(\begin{array}{ccc}
1 & \ell_{1} & \ell_{2} \\
0 & 1 & 0 \\
0 & 0 & 1
\end{array}\right)\left(\begin{array}{ccc}
2^{2 j} & 0 & 0 \\
0 & 2^{j} & 0 \\
0 & 0 & 2^{j}
\end{array}\right)=Q\left(\begin{array}{ccc}
2^{2 j} & 2^{j} \ell_{1} & 2^{j} \ell_{2} \\
0 & 2^{j} & 0 \\
0 & 0 & 2^{j}
\end{array}\right)
$$

Hence $T_{(j, \ell)}$ maps $\left(\xi_{1}, \xi_{2}, \xi_{3}\right)$ into $\left(2^{2 j} \xi_{1}, 2^{j}\left(\ell_{1} \xi_{1}+\xi_{2}\right), 2^{j}\left(\ell_{2} \xi_{1}+\xi_{3}\right)\right)$. In particular, the coordinate $\xi_{1}$ ranges over $[1 / 16,9 / 16]$ and under the action of $T_{(j, \ell)}$ this interval is mapped into $\left[2^{2 j-4}, 92^{2 j-4}\right]$. As inthe argument of Proposition 4.1, two such intervals can intersect each other only if $j-j^{\prime} \in\{0, \pm 1\}$. From now on, let $j, \ell$ be fixed. The tile $Q_{(j, \ell)}$ has the same $\xi_{2}$ and $\xi_{3}$ extension independently from $\ell$. Specifically, that value is $32^{j-3}$ on the left and $112^{j-3}$ on the right. We have that

$$
B_{(1)}^{[\ell]} A_{(1)}^{j}=\left(\begin{array}{ccc}
1 & \ell_{1} & \ell_{2} \\
0 & 1 & 0 \\
0 & 0 & 1
\end{array}\right)\left(\begin{array}{ccc}
2^{2 j} & 0 & 0 \\
0 & 2^{j} & 0 \\
0 & 0 & 2^{j}
\end{array}\right)=\left(\begin{array}{ccc}
2^{2 j} & 0 & 0 \\
0 & 2^{j} & 0 \\
0 & 0 & 2^{j}
\end{array}\right)\left(\begin{array}{ccc}
1 & 2^{-j} \ell_{1} & 2^{-j} \ell_{2} \\
0 & 1 & 0 \\
0 & 0 & 1
\end{array}\right) .
$$


Hence, for a fixed value of $\xi_{1}=\overline{\xi_{1}}$, the shear matrix produces a displacement by $2^{-j} \ell_{1} \xi_{1}$ and $2^{-j} \ell_{2} \xi_{1}$ in the directions $\xi_{2}$ and $\xi_{3}$, respectively. Further, at $\xi_{1}=2^{2 j^{\prime}-4}$ (the left bound of $Q_{\left(j^{\prime}, \ell^{\prime}\right)}$ ), the trapezoid $Q_{(j, \ell)}$ has height and depth equal to $2^{-j+2 j^{\prime}-3}+2^{j-2}$, while each time we apply $B_{(1)}^{[(1,0)]}$ or of $B_{(1)}^{[(0,1)]}$ the trapezoid $Q_{\left(j^{\prime}, \ell^{\prime}\right)}$ is displaced by $2^{j^{\prime}-4}$ along the $\xi_{2}$ or $\xi_{3}$ axes, respectively. Hence, using a computation similar to Proposition 4.1 we find that with respect to each directions there are at most 46 intersections. Hence, the number of total intersections is bounded by a constant independent of $j, \ell$.

It remains to prove property (2.2). That is, we need to show that there exists a constant $K>0$ such that if $Q_{(j, \ell)} \cap Q_{\left(j^{\prime}, \ell^{\prime}\right)} \neq \emptyset$ then $\left\|B_{(1)}^{[\ell]} A_{(1)}^{j} A_{(1)}^{-j^{\prime}} B_{(1)}^{\left[\ell^{\prime}\right]}\right\|_{\infty} \leq K$, or equivalently $\left\|B_{(1)}^{[\ell]} A_{(1)}^{j} A_{(1)}^{-j^{\prime}} B_{(1)}^{\left[\ell^{\prime}\right]}\right\|_{\ell^{1}} \leq K$. Computing explicitly the $\ell^{1}$ norm we obtain

$$
\begin{gathered}
\left\|B_{(1)}^{[\ell]} A_{(1)}^{j} A_{(1)}^{-j^{\prime}} B_{(1)}^{\left[\ell^{\prime}\right]}\right\|_{\ell^{1}}=\left\|\left(\begin{array}{ccc}
2^{2\left(j-j^{\prime}\right)} & 2^{j-j^{\prime}} \ell_{1}-2^{2\left(j-j^{\prime}\right)} \ell_{1}^{\prime} & 2^{j-j^{\prime}} \ell_{2}-2^{2\left(j-j^{\prime}\right)} \ell_{2}^{\prime} \\
0 & 2^{j-j^{\prime}} & 0 \\
0 & 0 & 2^{j-j^{\prime}}
\end{array}\right)\right\|_{\ell^{1}} \\
=\max \left\{2^{2\left(j-j^{\prime}\right)},\left|2^{j-j^{\prime}} \ell_{1}-2^{2\left(j-j^{\prime}\right)} \ell_{1}^{\prime}\right|+2^{j-j^{\prime}},\left|2^{j-j^{\prime}} \ell_{2}-2^{2\left(j-j^{\prime}\right)} \ell_{2}^{\prime}\right|+2^{j-j^{\prime}}\right\} .
\end{gathered}
$$

Observe that $Q T_{(j, \ell)} \cap Q T_{\left(j^{\prime}, \ell^{\prime}\right)} \neq \emptyset$ if and only if $Q T_{(j, \ell)} T_{\left(j^{\prime}, \ell^{\prime}\right)}^{-1} \cap Q \neq \emptyset$ and that, if this happens, then also the projections on the $\xi_{2}$ and $\xi_{3}$ axes must intersect. Write the different cases we obtain equations (4.19) where $\ell, \ell^{\prime}$ are replaced by $\ell_{1}, \ell_{1}^{\prime}$ and $\ell_{2}, \ell_{2}^{\prime}$. Hence we can repeat the argument of Proposition 4.1 obtaining exactly the same estimates:

$$
\left|\ell_{i} 2^{j-j^{\prime}}-\ell_{i}^{\prime} 2^{2\left(j-j^{\prime}\right)}\right| \leq 9
$$

for $i=1,2$. This implies that

$$
\left\|B_{(1)}^{[\ell]} A_{(1)}^{j} A_{(1)}^{-j^{\prime}} B_{(1)}^{\left[\ell^{\prime}\right]}\right\|_{\ell^{1}} \leq \max \left\{2^{2}, 9+2,9+2\right\} \leq 11 .
$$

We will refer to the structured admissible covering in Prop. 5.1 as the 3d shearlet-type covering. The following observations can be derived exactly as in the 2-dimensional case.

- The trapezoidal region

$$
P^{\prime}=\left\{\left(\xi_{1}, \xi_{2}\right):\left|\xi_{2}\right| \leq \xi_{1},\left|\xi_{3}\right| \leq \xi_{1}, \xi_{1} \in[1 / 8,1 / 2]\right\}
$$

gives rise to a minimal admissible covering of $\mathbb{R}^{3}$ with respect to the family of transformations $\mathcal{T}_{\mathcal{M}}$, where $\mathcal{T}_{\mathcal{M}}$ is given by (5.34), and it is equivalent to every possible structured admissible covering with respect to the same family of transformation.

- The $3 \mathrm{~d}$ curvelet-type (defined similarly to the $2 \mathrm{~d}$ case) and the shearlet-type coverings in $\mathbb{R}^{3}$ are equivalent.

We can now define the decomposition spaces associated with the admissible structured covering constructed above. Namely, we can define the decomposition space $D\left(\mathcal{Q}, L^{p},\left(\ell_{q}\right)_{2^{\beta}}\right)$, where $\mathcal{Q}$ is the shearlettype covering of $\mathbb{R}^{3}$ and $2^{\beta}$ is the $\mathcal{Q}$-moderate weight defined by $\left(2^{\beta}\right)_{(j, \ell, k, d)}=2^{j \beta}$. Also in this case, an argument similar to the one used in Sec. 4.5 can be used to provide a characterization of this smoothness spaces in terms of the shearlet coefficients.

Acknowledgements $\quad$ D. L. and P. N. were partially supported by NSF grants DMS 1008900 and DMS 1005799. This work was initiated while D.L. was visiting the NuHAG in Vienna. A part of this work was performed while L. M. was visiting the Department of Mathematics at the University of Houston. 


\section{References}

[1] L. Borup and M. Nielsen. Nonlinear approximation in $\alpha$-modulation spaces. Math. Nachr., 279:101-120, 2006.

[2] L. Borup and M. Nielsen. Frame decomposition of decomposition spaces. J. Fourier Anal. Appl., 13:39$70,2007$.

[3] E. J. Candès and D. L. Donoho. New tight frames of curvelets and optimal representations of objects with $C^{2}$ singularities. Comm. Pure Appl. Math., 57:219-266, 2004.

[4] P. G. Casazza. The art of frame theory. Taiwanese J. Math., 4:129-201, 2000.

[5] C. Chesneau, M.J. Fadili and J.-L. Starck. Stein block thresholding for image denoising. Appl. Comput. Harmon. Anal., 28(1):67-88, 2010.

[6] O. Christensen. An Introduction to Frames and Riesz Bases. Birkhäuser, Boston, 2003.

[7] S. Dahlke, G. Kutyniok, P. Maass, C. Sagiv, H.-G. Stark and G. Teschke. The uncertainty principle associated with the continuous shearlet transform. Int. J. Wavelets Multiresolut. Inf. Process., 6:157$181,2008$.

[8] S. Dahlke, G. Kutyniok, G. Steidl and G. Teschke. Shearlet coorbit spaces and associated Banach frames. Appl. Comput. Harmon. Anal., 27(2):195-214, 2009.

[9] S. Dahlke, G. Steidl and G. Teschke. Shearlet coorbit spaces: compactly supported analyzing shearlets, traces and embeddings. J. Fourier Anal. Appl., 17(6):1232-1255, 2011.

[10] S. Dahlke, G. Steidl and G. Teschke. Multivariate shearlet transform, shearlet coorbit spaces and their structural properties. In: Shearlets: Multiscale Analysis for Multivariate Data. G. Kutyniok and D. Labate, Birkhäuser, Boston, 2012.

[11] G. R. Easley, D. Labate and W. Lim. Sparse directional image representations using the discrete shearlet transform. Appl. Comput. Harmon. Anal., 25(1):25-46, 2008.

[12] H. G. Feichtinger. Banach spaces of distributions defined by decomposition methods, II. Math. Nachr., 132:207-237, 1987.

[13] H. G. Feichtinger and P. Gröbner. Banach spaces of distributions defined by decomposition methods, I. Math. Nachr., 123:97-120, 1985.

[14] H. G. Feichtinger and K. Gröchenig. Banach spaces related to integrable group representations and their atomic decomposition I. J. Funct. Anal., 86:307-340, 1989.

[15] H. G. Feichtinger and K. Gröchenig. Banach spaces related to integrable group representations and their atomic decomposition II. Monatsh. Math., 108:129-148, 1989.

[16] K. Gröchenig and S. Samarah. Nonlinear approximation with local Fourier bases. Constr. Approx., 16(3):317-331, 2000.

[17] P. Grohs and G. Kutyniok. Parabolic Molecules. (preprint) 2012.

[18] K. Guo and D. Labate. Optimally sparse multidimensional representation using shearlets. Siam J. Math. Anal., 9:298-318, 2007

[19] K. Guo and D. Labate. Representation of Fourier integral operators using shearlets. J. Fourier Anal. Appl., 14:327-371, 2008. 
[20] K. Guo and D. Labate. Optimally sparse representations of 3D data with $C^{2}$ surface singularities using Parseval frames of shearlets. Siam J. Math. Anal., 44:851-886 2012.

[21] K. Guo, and D. Labate. The construction of smooth Parseval frames of shearlets. Math. Model. Nat. Phenom. to appear, 2013.

[22] K. Guo, G. Kutyniok and D. Labate. Sparse multidimensional representations using anisotropic dilation and shear operators. In: Wavelets and Splines, G. Chen and M. Lai (eds.), Nashboro Press, Nashville, TN, 2006.

[23] G. Kutyniok and D. Labate. Resolution of the wavefront set using continuous shearlets. Trans. Amer. Math. Soc., 361:2719-2754, 2009.

[24] G. Kutyniok and D. Labate. Shearlets: Multiscale Analysis for Multivariate Data. Birkhäuser, Boston, 2012.

[25] Y. Meyer. Wavelets and Operators. Cambridge Stud. Adv. Math. vol. 37, Cambridge Univ. Press, Cambridge, UK, 1992.

[26] V. M. Patel, G. Easley and D.M. Healy. Shearlet-based deconvolution. IEEE Transactions on Image Processing, 18:2673-2685, 2009.

[27] K. N. Rasmussen and M. Nielsen. Compactly supported frames for decomposition spaces. J. Fourier Anal. Appl., 18:87-117, 2012.

[28] H. Triebel. Theory of Function Spaces. Birkhäuser, Basel, 1983.

[29] D. Vera. Triebel-Lizorkin spaces and shearlets on the cone in $\mathbb{R}^{2}$. Appl. Computat. Harmon. Anal., to appear, 2013. 